\title{
Sensor-Based Robust Incremental Three-Dimensional Guidance Law with Terminal Angle Constraint
}

\author{
Tuo Han* and Qinglei Hu ${ }^{\dagger}$ \\ Beihang University, 100191 Beijing, People's Republic of China \\ Hyo-Sang Shin ${ }^{\ddagger} \odot$ and Antonios Tsourdos ${ }^{\S}$ \\ Cranfield University, Cranfield, England MK43 OAL, United Kingdom \\ and \\ Ming Xin \\ University of Missouri, Columbia, Missouri 65211 \\ https://doi.org/10.2514/1.G006038
}

\begin{abstract}
In this work, a robust incremental three-dimensional (3D) guidance law is proposed considering terminal angle constraint against maneuvering targets. As a stepping stone, the line-of-sight (LOS) tracking error dynamics is employed for the 3D guidance law design. A sliding variable is constructed such that its first-order derivative excludes the relative range in the perturbation, which avoids the unboundedness of system perturbation induced by target maneuvers near collision. A time-varying version of the sliding variable is designed to accelerate convergence of the LOS tracking errors and avoid large initial sliding variables. Then, two guidance laws are derived as a benchmark via the nonlinear dynamic inversion (NDI)-based sliding mode control (NDI-SMC) and NDI-based time-varying sliding mode control (NDI-TVSMC), respectively. To further improve guidance robustness with reduced system perturbation, the sensor-based incremental nonlinear dynamic inversion (INDI) control is used to design the INDI-SMC-based and INDI-TVSMC-based guidance laws. The sensor-based guidance laws exploit the LOS angular acceleration and guidance command output at the latest step, which result in smaller guidance gains to reject the perturbation than the NDI guidance laws. Numerical simulations in various cases and comparison studies are conducted to verify the effectiveness and robustness of the proposed method.
\end{abstract}

\section{Introduction}

G UIDANCE system has been playing a crucial role in generating feasible trajectories for missiles against various target motions $[1,2]$ and planetary powered descent trajectory design [3]. The primary aim of designing guidance laws, e.g., the well-known proportional navigation guidance (PNG) laws, is to achieve the minimal miss distance [4,5]. Besides the precise interception mission, it is of special significance for investigating advanced guidance laws with terminal angle constraint near target [6-9]. Specifically, to maximize the warhead lethality and improve missile survivability, the impact angle constraint is required for missiles against enhanced protections (such as harder armor) on tanks and advanced defense systems (such as the close-in weapon system and electronic counter measure system) on warships [6,7]. To achieve desired approaching direction or obstacle avoidance, the terminal approach angle constraint can be imposed on missiles and autonomous vehicles $[8,9]$.

In recent years, the terminal angle constrained guidance problem has been widely investigated in the aerospace community. For instance, Ref. [6] proposed an impact angle guidance law via solving a linear quadratic optimal control problem. The desired impact angle was achieved in [7] for intercepting stationary targets via the optimal

Received 5 March 2021; revision received 24 May 2021; accepted for publication 25 May 2021; published online 22 July 2021. Copyright $@ 2021$ by the American Institute of Aeronautics and Astronautics, Inc. All rights reserved. All requests for copying and permission to reprint should be submitted to CCC at www.copyright.com; employ the eISSN 1533-3884 to initiate your request. See also AIAA Rights and Permissions www.aiaa.org/ randp.

*Ph.D. Student, School of Automation Science and Electrical Engineering; hantuo@buaa.edu.cn.

${ }^{\dagger}$ Professor, School of Automation Science and Electrical Engineering; huql_buaa@buaa.edu.cn. Associate Fellow AIAA (Corresponding Author).

Professor, School of Aerospace, Transport and Manufacturing; h.shin@ cranfield.ac.uk. Member AIAA.

${ }^{\S}$ Professor, School of Aerospace, Transport and Manufacturing; a.tsourdos@cranfield.ac.uk. Senior Member AIAA.

TProfessor, Department of Mechanical and Aerospace Engineering; xin@missouri.edu. Associate Fellow AIAA. control theory. To obtain the shortest distance for obstacle avoidance, zero yaw angles near the passing waypoint were considered in [8]. To achieve the desired approach velocity direction at a desired time, a hybrid guidance scheme was introduced in [9]. The PNG law was combined with an orientation strategy [10] and modified with biased forms $[11,12]$ to meet the desired impact angle. To intercept maneuvering targets with constrained terminal angle, the sliding mode control (SMC) was used to derive the guidance law for enhancing system robustness [14-19].

Note that these terminal angle constrained guidance laws are developed in a planar case, which assumes decoupled three-dimensional (3D) engagement dynamics. However, a real 3D interception is more practical for guidance law design, which attracts the recent study on the design of 3D guidance laws [20-30]. Thus, it is of practical significance to investigate the terminal angle constrained guidance law in the $3 \mathrm{D}$ space. There have been several 3D guidance laws considering the terminal angle constraint. In [23], a trajectory shaping 3D guidance law with impact angle constraint was proposed via developing reference line-of-sight (LOS) profiles. Then, adaptive multivariable twisting and super-twisting controls were employed to handle the impact angle constrained 3D guidance problems in [24,25]. In [26,27], the 3D guidance law was derived via the Lyapunov-based approach and SMC with dual sliding surfaces. Using the rotation angle and Euler axis of quaternion, the impact angle constraint was achieved in a $3 \mathrm{D}$ space in [28]. The impact angle constrained 3D guidance law was developed based on the SMC method in $[29,30]$.

It is worth mentioning that the problem of advanced guidance law design with terminal angle constraint remains open in three aspects:

1) Most of the above guidance laws are designed for stationary targets or in a planar case, which will hinder its application to maneuvering targets in the 3D space. The terminal angle constrained $3 \mathrm{D}$ guidance law considering maneuvering targets can be found in $[24,29,30]$.

2) The lumped perturbation including the target maneuvers or system uncertainties for guidance law designs in [15,20,24,25,29] increases with the decreased relative range. This is because the relative range takes part in the denominator of the unknown perturbation. Although the gain adaption and disturbance observer techniques are useful tools to compensate such unknown perturbations

Published by AIAA. This is the Author Accepted Manuscript issued with: Creative Commons Attribution Non-Commercial License (CC:BY:NC 4.0).

The final published version (version of record) is available online at DOI:10.2514/1.G006038. Please refer to any applicable publisher terms of use. 
([24,29], to name a few), the fact that the perturbation becomes large if the relative range is near zero and even infinite at the collision point (relative range is zero) is still true. This issue would lead to the difficulty of achieving desired terminal angles at the collision point because of the limited perturbation rejection ability, which results from the third aspect:

3) Most of the above robust terminal angle constrained guidance laws are based on the condition that the perturbation cancellation gains are greater than the upper bound of the perturbation. This means that higher gains are required when the perturbation (such as the target maneuver) increases. The disadvantages of involving high gains include the following: a) large guidance commands will be created; b) undesirable chattering issue will occur for SMC-based guidance laws.

Motivated by the above discussions, this work aims to resolve the aforementioned three issues simultaneously. The terminal angle constrained 3D guidance law against maneuvering targets is designed by removing the relative range from the lumped perturbation and reducing the upper bound of the lumped perturbation. Specifically, a sliding variable is constructed such that the lumped perturbation existing in the sliding dynamics is not related to the relative range. Then, the nonlinear dynamic inversion (NDI)-based SMC (NDI-SMC) and NDI-based time-varying sliding mode control (NDI-TVSMC) are developed to construct the 3D guidance law. To reduce the upper bound of the lumped perturbation and improve system robustness, the idea of combining sensor-based incremental nonlinear dynamic inversion control (INDI) and SMC (named INDI-SMC [31-33]) that inherits the merits and avoids the defects of both methods is employed to design the INDI-SMC and INDI-TVSMC 3D guidance laws. To the best of our knowledge, this is the first paper developing the incremental guidance law. The main contributions are as follows:

1) The 3D guidance law with terminal angle constraint against maneuvering targets is designed without introducing unbounded perturbation induced by target maneuvers near collision.

2) The sensor-based incremental control concept is combined with the SMC and TVSMC for guidance law design, which reduces the upper bound of the unknown lumped perturbation and enhances the guidance system robustness simultaneously.

3) Under the bounded target maneuvers, the unknown lumped perturbation can be rejected by INDI-SMC-based and INDITVSMC-based guidance laws using smaller gains than that by NDI-SMC-based and NDI-TVSMC-based guidance laws.

The rest of this paper is organized as follows. The problem is described in Sec. II. The guidance law design and analysis are presented in Sec. III. Simulation results are shown in Sec. IV, and concluding remarks are given in Sec. V.

\section{Problem Description}

In this section, the nonlinear 3D guidance model for the missile and target is presented, and the guidance mission in this work is described in detail.

\section{A. Three-Dimensional Interception Kinematics and Dynamics}

The 3D homing interception geometry for the missile (denoted as $M$ ) and target (denoted as $T$ ) is shown in Fig. 1. Based on the Cartesian inertial reference frame $O X_{I} Y_{I} Z_{I}$, the missile and target body coordinates as well as the LOS frame are described by $O X_{M} Y_{M} Z_{M}, O X_{T} Y_{T} Z_{T}$, and $O X_{L} Y_{L} Z_{L}$, respectively. The LOS angles, velocity lead angles, and flight path angles of the missile are represented as $\theta_{L}, \phi_{L}, \theta_{M}, \phi_{M}$, and $\gamma_{M}, \vartheta_{M}$, respectively. The velocity lead angles, and flight path angles of the target are represented as $\theta_{T}, \phi_{T}$, and $\gamma_{T}, \vartheta_{T}$, respectively. The missile and target's speeds are denoted by $V_{M}$ and $V_{T}$, respectively. The relative range between the missile and target is $r$.

The 3D interception kinematics and dynamics can be given as follows [34]:

$$
\begin{gathered}
\dot{r}=V_{T} \cos \theta_{T} \cos \phi_{T}-V_{M} \cos \theta_{M} \cos \phi_{M} \\
r \dot{\theta}_{L}=V_{T} \sin \theta_{T}-V_{M} \sin \theta_{M}
\end{gathered}
$$

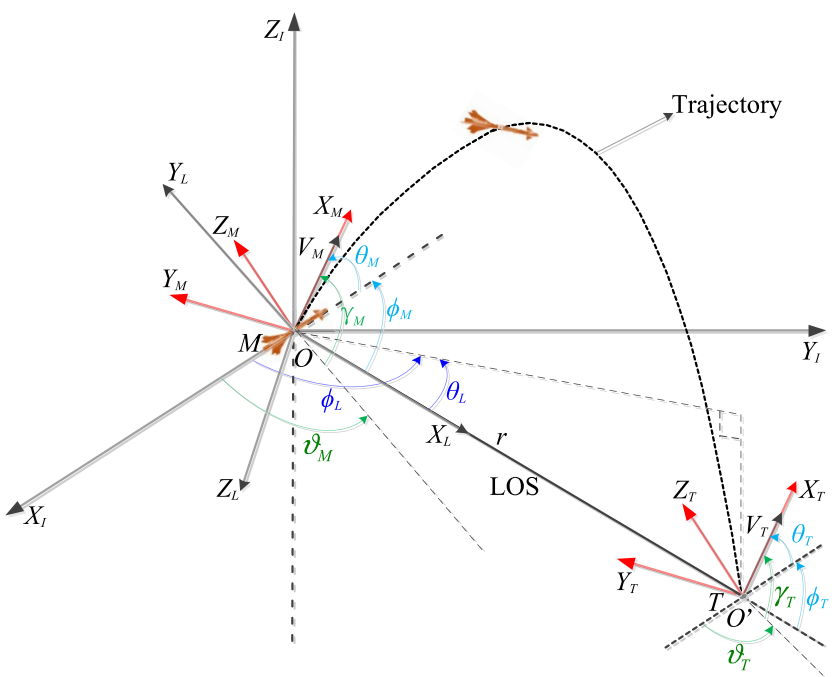

Fig. 1 Three-dimensional interception geometry for the missile and target.

$$
\begin{gathered}
r \dot{\phi}_{L} \cos \theta_{L}=V_{T} \cos \theta_{T} \sin \phi_{T}-V_{M} \cos \theta_{M} \sin \phi_{M} \\
\dot{\theta}_{M}=A_{z M} / V_{M}-\cos \phi_{M} \dot{\theta}_{L}-\sin \theta_{L} \sin \phi_{M} \dot{\phi}_{L} \\
\dot{\phi}_{M}=\frac{A_{y M}}{V_{M} \cos \theta_{M}}-\tan \theta_{M} \sin \phi_{M} \dot{\theta}_{L}-\dot{\phi}_{L} \cos \theta_{L} \\
\quad+\sin \theta_{L} \tan \theta_{M} \cos \phi_{M} \dot{\phi}_{L} \\
\dot{\theta}_{T}=A_{z T} / V_{T}-\cos \phi_{T} \dot{\theta}_{L}-\sin \theta_{L} \sin \phi_{T} \dot{\phi}_{L} \\
\dot{\phi}_{T}= \\
\quad \frac{A_{y T}}{V_{T} \cos \theta_{T}}-\tan \theta_{T} \sin \phi_{T} \dot{\theta}_{L}-\dot{\phi}_{L} \cos \theta_{L} \\
\quad+\sin \theta_{L} \tan \theta_{T} \cos \phi_{T} \dot{\phi}_{L}
\end{gathered}
$$

where $A_{z M}, A_{y M}$ and $A_{z T}, A_{y T}$ denote the missile and target's normal accelerations in the pitch and yaw planes, respectively. The inertial positions of the missile and target (denoted as $x_{M}, y_{M}, z_{M}$ and $x_{T}, y_{T}$, $z_{T}$ ) are governed by

$$
\left[\begin{array}{c}
\dot{x}_{M} \\
\dot{y}_{M} \\
\dot{z}_{M} \\
\dot{\gamma}_{M} \\
\dot{\vartheta}_{M}
\end{array}\right]=\left[\begin{array}{c}
V_{M} \cos \gamma_{M} \cos \vartheta_{M} \\
V_{M} \cos \gamma_{M} \sin \vartheta_{M} \\
V_{M} \sin \gamma_{M} \\
A_{z M} / V_{M} \\
A_{y M} /\left(V_{M} \cos \gamma_{M}\right)
\end{array}\right],\left[\begin{array}{c}
\dot{x}_{T} \\
\dot{y}_{T} \\
\dot{z}_{T} \\
\dot{\gamma}_{T} \\
\dot{\vartheta}_{T}
\end{array}\right]=\left[\begin{array}{c}
V_{T} \cos \gamma_{T} \cos \vartheta_{T} \\
V_{T} \cos \gamma_{T} \sin \vartheta_{T} \\
V_{T} \sin \gamma_{T} \\
A_{z T} / V_{T} \\
A_{y T} /\left(V_{T} \cos \gamma_{T}\right)
\end{array}\right]
$$

\section{B. Guidance Objectives}

This work aims to achieve the following guidance objectives:

Zero miss distance: The missile should successfully intercept the target, i.e., $r \rightarrow 0$.

Terminal angle constraint: The missile needs to achieve desired terminal LOS angle constraints at the collision point. Specifically, the LOS angles should meet $\theta_{L}, \phi_{L} \rightarrow \theta_{L F}, \phi_{L F}\left(\theta_{L F}, \phi_{L F}\right.$ are the expected terminal LOS angles) to achieve the impact angle constraint for non-maneuvering targets because there exists a specific relation between them [19]. For maneuvering targets, because their maneuvers are unknown in this work, the desired terminal LOS angle refers to the approach angle constraint instead of the impact angle constraint [24].

Guidance robustness: The guidance system is robust to the lumped perturbation (including the system uncertainties and target maneuvers) 
whose upper bound is reduced and the unboundedness near collision is avoided.

Remark 1: The third objective is a challenging task for guidance law design against maneuvering targets based on the LOS dynamics. Specifically, the perturbation induced by target maneuvers is usually presented in the form of $h\left(A_{z, y T}\right) / r$ ([20,24,25], to name a few), where $h\left(A_{z, y T}\right)$ is a function of the target acceleration. It is obvious that $h\left(A_{z, y T}\right) / r$ will diverge if the relative range goes near zero (depending on the minimum miss distance requirement). Especially, this term becomes infinite when the relative range is zero. This results in limited perturbation rejection ability of the guidance system, and consequently inaccurate terminal angles at the collision point will occur.

\section{Sensor-Based Incremental Guidance Law Design}

To achieve the above guidance objectives, a 3D guidance law is first designed as a benchmark via the NDI-based SMC technique. The convergence rate of LOS tracking errors is accelerated by extending the sliding variable to a time-varying version. Then, the sensor-based incremental guidance law is designed based on the combination of the INDI and SMC methods. Finally, the NDI-SMC-based, NDI-TVSMC-based, INDI-SMC-based, and INDI-TVSMC-based guidance laws are compared.

\section{A. LOS Tracking Error Dynamics}

The nonlinear coupled LOS dynamics can be obtained from Eqs. (2-7) as [30]

$$
\begin{aligned}
\ddot{\theta}_{L}= & -\frac{2 \dot{r} \dot{\theta}_{L}}{r}-\frac{\cos \theta_{M}}{r} A_{z M}+\frac{\cos \theta_{T}}{r} A_{z T}-\dot{\phi}_{L}^{2} \sin \theta_{L} \cos \theta_{L} \\
\ddot{\phi}_{L}= & -\frac{2 \dot{r} \dot{\phi}_{L}}{r}+\frac{\sin \theta_{M} \sin \phi_{M}}{r \cos \theta_{L}} A_{z M}-\frac{\cos \phi_{M}}{r \cos \theta_{L}} A_{y M} \\
& -\frac{\sin \theta_{T} \sin \phi_{T}}{r \cos \theta_{L}} A_{z T}+\frac{\cos \phi_{T}}{r \cos \theta_{L}} A_{y T}+2 \dot{\phi}_{L} \dot{\theta}_{L} \tan \theta_{L}
\end{aligned}
$$

Define the LOS tracking errors as $e_{1}=\theta_{L}-\theta_{L F}, e_{2}=\phi_{L}-\phi_{L F}$, and the error dynamics can be presented as

$$
\left\{\begin{array}{l}
\dot{\boldsymbol{e}}_{\mathrm{LOS}}=\boldsymbol{e}_{\mathrm{Rate}} \\
\dot{\boldsymbol{e}}_{\mathrm{Rate}}=\boldsymbol{A}+\boldsymbol{B} \boldsymbol{U}+\boldsymbol{D}
\end{array}\right.
$$

where $\boldsymbol{e}_{\mathrm{LOS}}=\left[e_{1}, e_{2}\right]^{T}, \quad \boldsymbol{U}=\left[A_{z M}, A_{y M}\right]^{T}, \quad \boldsymbol{e}_{\text {Rate }}=\left[\dot{e}_{1}, \dot{e}_{2}\right]^{T}=$ $\left[\dot{\theta}_{L}-\dot{\theta}_{L F}, \dot{\phi}_{L}-\dot{\phi}_{L F}\right]^{T}$, and

$$
\begin{aligned}
& \boldsymbol{A}=\left[\begin{array}{cc}
-\frac{2 \dot{r} \dot{\theta}_{L}}{r}-\frac{1}{2} \dot{\phi}_{L}^{2} \sin 2 \theta_{L}-\ddot{\theta}_{L F} \\
-\frac{2 \dot{\phi} \dot{\phi}_{L}}{r}+2 \dot{\phi}_{L} \dot{\theta}_{L} \tan \theta_{L}-\ddot{\phi}_{L F}
\end{array}\right], \\
& \boldsymbol{B}=\left[\begin{array}{cc}
\frac{-\cos \theta_{M}}{r} & 0 \\
\frac{\sin \theta_{M} \sin \phi_{M}}{r \cos \theta_{L}} & \frac{-\cos \phi_{M}}{r \cos \theta_{L}}
\end{array}\right], \\
& \boldsymbol{D}=\left[\begin{array}{c}
A_{z T} \cos \theta_{T} / r \\
\left(\cos \phi_{T} A_{y T}-\sin \theta_{T} \sin \phi_{T} A_{z T}\right) /\left(r \cos \theta_{L}\right)
\end{array}\right]
\end{aligned}
$$

Assumption 1 [26]: The velocity lead angles are assumed to satisfy $\left|\theta_{M}\right| \neq \pi / 2$ and $\left|\phi_{M}\right| \neq \pi / 2$, and the LOS angle $\left|\theta_{L}\right|=\pi / 2$ that performs a high diving maneuver is not considered.

$\dot{\theta}_{L F}, \dot{\phi}_{L F}, \ddot{\theta}_{L F}, \ddot{\phi}_{L F}$ are the first-order and second-order derivatives of the desired LOS angles, respectively. Because the desired LOS angles are constant values, $\dot{\theta}_{L F}=\dot{\phi}_{L F}=\ddot{\theta}_{L F}=\ddot{\phi}_{L F}=0$ holds in this work. $\boldsymbol{D}$ is the perturbation induced by the unknown target maneuvers. Note that the perturbation will increase dramatically and become infinite as the relative range decreases towards zero. The guidance law design directly based on Eq. (11) requires adaptive control or disturbance observer techniques to compensate for the large perturbations near collision.

\section{B. NDI-SMC Guidance Law}

To avoid the unbounded perturbation $\boldsymbol{D}$ near collision, the following sliding variable (denoted as $\boldsymbol{s}$ ) is designed:

$$
\boldsymbol{s}=\boldsymbol{r} \boldsymbol{e}_{\mathrm{Rate}}+k \boldsymbol{e}_{\mathrm{LOS}}
$$

where $k$ is a positive parameter to be designed.

Taking the first-order derivative of Eq. (12) leads to

$$
\dot{\boldsymbol{s}}=\dot{\boldsymbol{r}} \boldsymbol{e}_{\text {Rate }}+r \dot{\boldsymbol{e}}_{\text {Rate }}+k \boldsymbol{e}_{\text {Rate }}
$$

Substituting Eq. (11) into Eq. (13) yields

$$
\dot{\boldsymbol{s}}=\dot{\boldsymbol{r}} \boldsymbol{e}_{\mathrm{Rate}}+r(\boldsymbol{A}+\boldsymbol{B} \boldsymbol{U}+\boldsymbol{D})+k \boldsymbol{e}_{\mathrm{Rate}}
$$

Rewriting Eq. (14) as

$$
\dot{\boldsymbol{s}}=\tilde{\boldsymbol{A}}+\tilde{\boldsymbol{B}} \boldsymbol{U}+\tilde{\boldsymbol{D}}
$$

where

$$
\begin{aligned}
& \tilde{\boldsymbol{A}}=(\dot{r}+k) \boldsymbol{e}_{\text {Rate }}+r \boldsymbol{A}=\left[\begin{array}{c}
(-\dot{r}+k) \dot{\theta}_{L}-\frac{1}{2} r \dot{\phi}_{L}^{2} \sin 2 \theta_{L} \\
(-\dot{r}+k) \dot{\phi}_{L}+2 r \dot{\phi}_{L} \dot{\theta}_{L} \tan \theta_{L}
\end{array}\right], \\
& \tilde{\boldsymbol{B}}=r \boldsymbol{B}=\left[\begin{array}{cc}
-\cos \theta_{M} & 0 \\
\frac{\sin \theta_{M} \sin \phi_{M}}{\cos \theta_{L}} & \frac{-\cos \phi_{M}}{\cos \theta_{L}}
\end{array}\right] \\
& \tilde{\boldsymbol{D}}=r \boldsymbol{D}=\left[\begin{array}{c}
A_{z T} \cos \theta_{T} \\
\left(\cos \phi_{T} A_{y T}-\sin \theta_{T} \sin \phi_{T} A_{z T}\right) / \cos \theta_{L}
\end{array}\right]
\end{aligned}
$$

Assumption 2: The target maneuvers are continuous and bounded such that $\tilde{\boldsymbol{D}}$ is bounded and satisfy $\|\tilde{\boldsymbol{D}}\| \leq L$ with a positive scalar $L$.

Remark 2: Assumption 2 is reasonable and more practical compared with the direct boundedness assumption on $\boldsymbol{D}$, because the relative range in the denominator of $\boldsymbol{D}$ is removed.

Note that $s=k e_{\text {LOS }}$ if $r=0$ as per Eq. (12), which means that the sliding variable does not make sense for guidance law design under this case. Therefore, $s=0$ should be achieved before collision. To ensure convergence of the sliding variable, the NDI-SMC-based guidance law can be designed from Eq. (15) as follows:

$$
\boldsymbol{U}_{\mathrm{NDI}-\mathrm{SMC}}=\boldsymbol{U}_{\mathrm{NDI}-\mathrm{SMC}}^{\mathrm{eq}}+\tilde{\boldsymbol{B}}^{-1} \boldsymbol{v}
$$

where $v=-\beta s /\|s\|$ is a vector to reject the perturbation $\tilde{D}, \beta$ is a positive parameter to be designed, and

$$
\boldsymbol{U}_{\mathrm{NDI}-\mathrm{SMC}}^{\mathrm{eq}}=-\tilde{\boldsymbol{B}}^{-1} \tilde{\boldsymbol{A}}
$$

Select a Lyapunov function as $W_{1}=1 / 2 \boldsymbol{s}^{T} \boldsymbol{s}$, then $\dot{W}_{1}=\boldsymbol{s}^{T}(\tilde{\boldsymbol{A}}+$ $\tilde{\boldsymbol{B}} \boldsymbol{U}+\tilde{\boldsymbol{D}})$. Substituting Eq. (16) into it yields

$$
\dot{W}_{1}=\boldsymbol{s}^{T}(-\beta \boldsymbol{s} /\|\boldsymbol{s}\|+\tilde{\boldsymbol{D}}) \leq(-\beta+L)\|\boldsymbol{s}\|<0
$$

Therefore, the sliding variable $s$ can converge to the origin if $\beta>L$. After $s$ reaches the condition $s=0$, it can be obtained from Eq. (12) that

$$
\boldsymbol{e}_{\text {Rate }}=-(k / r) \boldsymbol{e}_{\mathrm{LOS}}
$$

which means

$$
\dot{e}_{1}=-(k / r) e_{1}, \quad \dot{e}_{2}=-(k / r) e_{2}
$$


Select a Lyapunov function as $W_{2}=1 / 2\left(e_{1}^{2}+e_{2}^{2}\right)$, and it can be derived from Eq. (20) that

$$
\dot{W}_{2}=e_{1} \dot{e}_{1}+e_{2} \dot{e}_{2}=-(k / r)\left(e_{1}^{2}+e_{2}^{2}\right)=-(2 k / r) W_{2}
$$

Because $k$ and $r$ are positive, the LOS tracking errors $e_{1}, e_{2}$ can converge to the origin exponentially. This also implies that $\theta_{L}$, $\phi_{L} \rightarrow \theta_{L F}, \phi_{L F}$, and $\dot{\theta}_{L}, \dot{\phi}_{L} \rightarrow \dot{\theta}_{L F}, \dot{\phi}_{L F}=0$. With the zero LOS rates, it can be known that zero miss distance can be finally achieved. Because the perturbation $\tilde{\boldsymbol{D}}$ will not diverge to undesirable values near collision such that $\|\tilde{\boldsymbol{D}}\|>L$, the condition $-\beta+L<0$ for Eq. (18) will be valid throughout the guidance process.

Remark 3: It should be noted from Eq. (20) that the convergence rate of $e_{1}, e_{2}$ is affected by the parameter $k$ and the relative range $r$. For example, because the relative range $r$ is large at the initial stage, a small $k$ would cause $e_{1} \neq 0, e_{2} \neq 0$ throughout the guidance process, which means $\dot{\theta}_{L}=\dot{e}_{1}=-(k / r) e_{1} \rightarrow \infty$ and $\dot{\phi}_{L}=\dot{e}_{2}=$ $-(k / r) e_{2} \rightarrow \infty$ as the relative range $r \rightarrow 0$. This implies that small $k$ will lead to LOS rate and guidance command divergence. One may suggest that selecting a large $k$ can ensure fast convergence of the LOS tracking errors. However, large $k$ will cause large initial sliding variables that require high gains of $\beta$ to eliminate, which will lead to undesirable chattering. This dilemma will be addressed in the next subsection.

\section{NDI-TVSMC Guidance Law}

To accelerate convergence rate of $e_{1}, e_{2}$ and avoid large initial sliding variables, the sliding variable in Eq. (12) is extended to a timevarying sliding variable as follows:

$$
\boldsymbol{s}_{v}=r \boldsymbol{e}_{\text {Rate }}+k_{v}(t) \boldsymbol{e}_{\mathrm{LOS}}
$$

where

$$
k_{v}(t)=k_{v 0}+\alpha\left(t-t_{0}\right)>0
$$

with $k_{v}\left(t_{0}\right)=k_{v 0}>0, \alpha$ is a positive constant, and $t \geq t_{0}$ is the flight time.

Taking the first-order derivative of Eq. (22) leads to

$$
\dot{\boldsymbol{s}}_{v}=r \dot{\boldsymbol{e}}_{\mathrm{Rate}}+\dot{\boldsymbol{r}} \boldsymbol{e}_{\mathrm{Rate}}+k_{v}(t) \boldsymbol{e}_{\mathrm{Rate}}+\dot{k}_{v}(t) \boldsymbol{e}_{\mathrm{LOS}}
$$

Substituting Eqs. (11) and (23) into Eq. (24) yields

$$
\dot{\boldsymbol{s}}_{v}=r \boldsymbol{A}+\boldsymbol{r} \boldsymbol{B} \boldsymbol{U}+\boldsymbol{r} \boldsymbol{D}+\dot{\boldsymbol{r}} \boldsymbol{e}_{\mathrm{Rate}}+k_{v}(t) \boldsymbol{e}_{\mathrm{Rate}}+\alpha \boldsymbol{e}_{\mathrm{LOS}}
$$

Rewriting Eq. (25) as

$$
\dot{\boldsymbol{s}}_{v}=\tilde{\boldsymbol{A}}_{v}+\tilde{\boldsymbol{B}} \boldsymbol{U}-\alpha \boldsymbol{C}+\tilde{\boldsymbol{D}}
$$

where $\boldsymbol{C}=\left[\theta_{L F}, \phi_{L F}\right]^{T}$, and

$$
\tilde{\boldsymbol{A}}_{v}=\left[\begin{array}{c}
\left(-\dot{r}+k_{v}\right) \dot{\theta}_{L}-\frac{1}{2} r \dot{\phi}_{L}^{2} \sin 2 \theta_{L}+\alpha \theta_{L} \\
\left(-\dot{r}+k_{v}\right) \dot{\phi}_{L}+2 r \dot{\phi}_{L} \dot{\theta}_{L} \tan \theta_{L}+\alpha \phi_{L}
\end{array}\right]
$$

Similar to the NDI-SMC-based guidance law in Eq. (16), the NDITVSMC-based guidance law can be designed as

$$
\boldsymbol{U}_{\mathrm{NDI}-\mathrm{TVSMC}}=\boldsymbol{U}_{\mathrm{NDI}-\mathrm{TVSMC}}^{\mathrm{eq}}+\tilde{\boldsymbol{B}}^{-1} \boldsymbol{v}_{v}
$$

where $\boldsymbol{v}_{v}=-\beta_{v} \boldsymbol{s}_{v} /\left\|\boldsymbol{s}_{v}\right\|$ with a positive parameter $\beta_{v}$, and

$$
\boldsymbol{U}_{\mathrm{NDI}-\mathrm{TVSMC}}^{\mathrm{eq}}=\tilde{\boldsymbol{B}}^{-1}\left(-\tilde{\boldsymbol{A}}_{v}+\alpha \boldsymbol{C}\right)
$$

Define a Lyapunov function as $W_{v}=1 / 2 \boldsymbol{s}_{v}^{T} \boldsymbol{s}_{v}$, then $\dot{W}_{v}=$ $\boldsymbol{s}_{v}^{T}\left(\tilde{\boldsymbol{A}}_{v}+\tilde{\boldsymbol{B}} \boldsymbol{U}-\alpha \boldsymbol{C}+\tilde{\boldsymbol{D}}\right)$. Substituting Eq. (27) into it leads to $\dot{W}_{v} \leq$ $\left(-\beta_{v}+L\right)\left\|\boldsymbol{s}_{v}\right\|$. Obviously, the sliding variable $\boldsymbol{s}_{v}$ can converge to the origin if $\beta_{v}>L$. When $\boldsymbol{s}_{v}=0$ is met, it can be obtained from Eq. (22) that

$$
\dot{e}_{1}=-\left(k_{v} / r\right) e_{1}, \quad \dot{e}_{2}=-\left(k_{v} / r\right) e_{2}
$$

Under the same $k_{v 0}$, the convergence rate of $e_{1}, e_{2}$ under Eq. (29) increases with a larger $\alpha$ selected in Eq. (23).

Compared with the NDI-SMC, the benefits of the NDI-TVSMC are as follows:

1) Under the same system initial conditions and $\beta=\beta_{v}$, the convergence rate of the sliding variable can be accelerated by selecting a small initial $k_{v 0}$ to meet a small initial sliding variable.

2) After the sliding variable reaches zero, the convergence rate of $e_{1}, e_{2}$ can be accelerated with the increased parameter $k_{v}$. Moreover, the convergence rate can be further enhanced with greater $\alpha$.

To better understand the convergence differences between the sliding variables $\boldsymbol{s}, \boldsymbol{s}_{v}$ and the tracking errors $e_{1}, e_{2}$, a set of illustrative examples is shown in Figs. 2-4. The initial conditions are set to $r_{0}=5000, e_{10}=60^{\circ}, e_{20}=-60^{\circ}, \dot{e}_{10}=-1 \times 10^{-3} \mathrm{rad} / \mathrm{s}$, and $\dot{e}_{20}=1 \times 10^{-3} \mathrm{rad} / \mathrm{s} ; \dot{r}=-10-40 t$ is selected. In Figs. 2 and 3, it can be seen that the SMC cannot enforce the tracking errors to zero under a small gain of $k=100$, despite that the convergence of the sliding variables is equivalent to that by TVSMC. By increasing $k$ and $\beta$, the system tracking errors can be driven to the origin by SMC. However, the initial sliding variable is greatly increased, which reduces the convergence rate of the sliding variables. At the same time, another issue caused by higher gains is the undesirable frequent

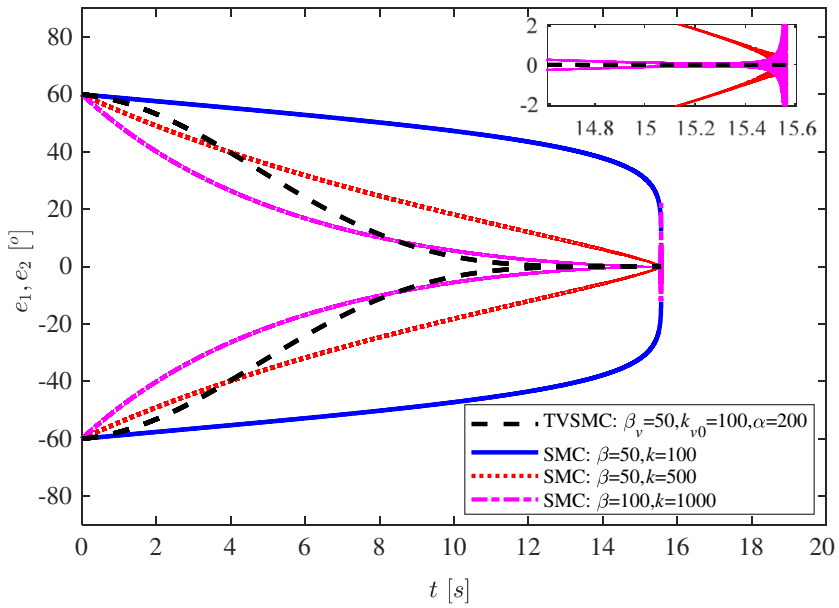

Fig. 2 Comparison of system tracking errors by SMC and TVSMC.

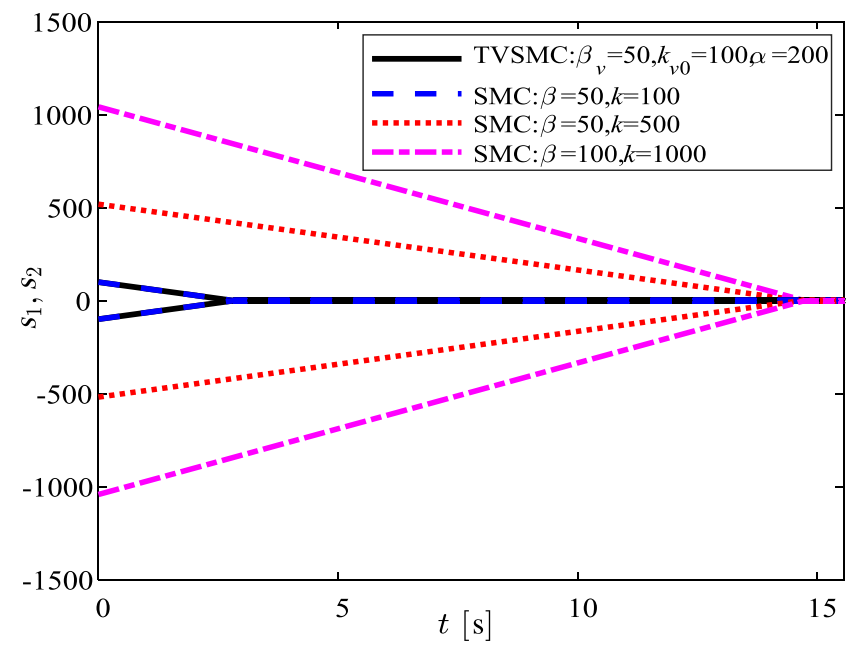

Fig. 3 Comparison of sliding variables by SMC and TVSMC. 


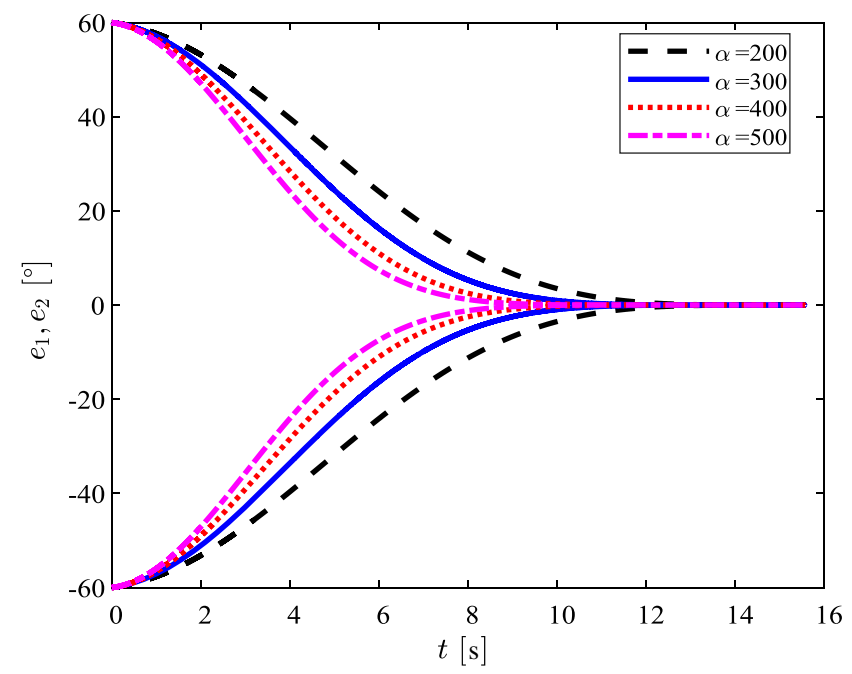

Fig. 4 Comparison of system tracking errors by TVSMC under $\beta_{v}=50, k_{v 0}=100$.

chattering. On the contrary, the TVSMC has faster convergence on the tracking errors than SMC and avoids large initial sliding variables. In Fig. 4, it can be seen that the system tracking errors under TVSMC can converge to zero faster by increasing $\alpha$.

\section{Sensor-Based Incremental Guidance Law}

In addition to the convergence rate improvement, the robustness issue still exists in the above guidance laws. Specifically, the above guidance laws require that the gains in $\boldsymbol{v}$ and $\boldsymbol{v}_{v}$ should satisfy $\beta>L$ and $\beta_{v}>L$, respectively. For some cases that the perturbation leads to a greater $L$, higher gains of $\beta$ and $\beta_{v}$ are needed. However, the disadvantage of introducing high gains in $\boldsymbol{v}$ and $\boldsymbol{v}_{v}$ is potential undesirable chattering. One may suggest that gain adaption methods for $\beta, \beta_{v}$ can be useful to compensate $\tilde{\boldsymbol{D}}$. Nevertheless, the adaption is to avoid gain overestimation, and the minimum values of $\beta, \beta_{v}$ should still be greater than $L$. Therefore, it is necessary to design the guidance law with reduced upper bound of the system perturbation, such that the robustness against target maneuvers can be enhanced and the chattering issue can be mitigated with smaller gains of $\beta, \beta_{v}$.

To achieve the aim, this paper leverages the sensor-based INDI method [31-33] that exploits the latest angular acceleration measurement and control output into the guidance law design. Specifically, the NDI-SMC-based and NDI-TVSMC-based guidance laws are extended to sensor-based INDI-SMC and INDI-TVSMC guidance laws, respectively.

Taking the design process of INDI-TVSMC guidance law as an example, the first step is to rewrite Eq. (26) as

$$
\dot{\boldsymbol{s}}_{v r}=\tilde{\boldsymbol{A}}_{v}(\boldsymbol{x})+\tilde{\boldsymbol{B}}(\boldsymbol{x}) \boldsymbol{U}+\boldsymbol{D}_{r}-\alpha \boldsymbol{C}
$$

where $\boldsymbol{s}_{v r}$ has the same form of $\boldsymbol{s}_{v}$ in Eq. (22), $\boldsymbol{x}$ represents the system state, and

$$
D_{r}=\tilde{D}+d
$$

where $\boldsymbol{d}$ is the unknown but bounded term caused by system uncertainties and external disturbances.

Then, the incremental dynamics of $\boldsymbol{s}_{v r}$ can be obtained by taking the first-order Taylor expansion of Eq. (30) around $t_{0}=t-\Delta t$ ( $\Delta t$ is the sampling interval) [31-33] as follows:

$$
\dot{\boldsymbol{s}}_{v r}=\dot{\boldsymbol{s}}_{v r 0}+\tilde{\boldsymbol{B}}_{0} \Delta \boldsymbol{U}+\Delta \boldsymbol{D}_{r}+\boldsymbol{\rho}(\boldsymbol{x})
$$

where $\dot{\boldsymbol{s}}_{v r 0}$ is the derivative of the sliding variable $\boldsymbol{s}_{v r}$ at $t_{0} . \Delta \boldsymbol{U}=$ $\boldsymbol{U}-\boldsymbol{U}_{0}$ is the increment of $\boldsymbol{U}$ from $t_{0}$ to $t . \Delta \boldsymbol{D}_{r}$ denotes the variations of the perturbation $\boldsymbol{D}_{r}$ over $\Delta t$, and $\tilde{\boldsymbol{B}}_{0}=\tilde{\boldsymbol{B}}\left(\boldsymbol{x}_{0}\right) \cdot \boldsymbol{\rho}(\boldsymbol{x})$ is defined as [31]

$$
\boldsymbol{\rho}(\boldsymbol{x})=\left.\frac{\partial\left[\tilde{\boldsymbol{A}}_{v}(\boldsymbol{x})+\tilde{\boldsymbol{B}}(\boldsymbol{x}) \boldsymbol{U}\right]}{\partial \boldsymbol{x}}\right|_{0} \Delta \boldsymbol{x}+\mathcal{O}\left(\Delta \boldsymbol{x}^{2}\right)
$$

where $\Delta \boldsymbol{x}=\boldsymbol{x}-\boldsymbol{x}_{0}$ is the increment of $\boldsymbol{x}$ from $t_{0}$ to $t$, and $\mathcal{O}\left(\Delta \boldsymbol{x}^{2}\right)$ includes the remaining higher-order terms.

Assumption 3 [32]: The partial derivatives of $\tilde{\boldsymbol{A}}, \tilde{\boldsymbol{A}}_{v}$, and $\tilde{\boldsymbol{B}}$ with respect to $\boldsymbol{x}$ are bounded for any order.

For the dynamics in Eq. (32), the equivalent incremental TVSMC guidance law can be designed as

$$
\Delta \boldsymbol{U}_{\text {INDI-TVSMC }}^{\mathrm{eq}}=-\tilde{\boldsymbol{B}}_{0}^{-1} \dot{\boldsymbol{s}}_{v r 0}
$$

Remark 4: Note that $\dot{\boldsymbol{s}}_{v r 0}$ can be estimated/measured because $\dot{\boldsymbol{s}}_{v r 0}=r_{0} \dot{\boldsymbol{e}}_{\text {Rate } 0}+\left(\dot{r}_{0}+k_{v 0}\right) \boldsymbol{e}_{\text {Rate } 0}+\alpha \boldsymbol{e}_{\mathrm{LOS} 0}$, where $k_{v 0}$ and $\alpha$ are known parameters, and $r_{0}, \dot{r}_{0}, \boldsymbol{e}_{\mathrm{Rate} 0}=\left[\dot{\theta}_{L 0}, \dot{\phi}_{L 0}\right]^{T}$, and $\boldsymbol{e}_{\mathrm{LOS} 0}=$ $\left[\theta_{L 0}-\theta_{L F}, \phi_{L 0}-\phi_{L F}\right]^{T}$ require the relative range, relative speed, LOS rates, and LOS angles, which are measurable by the onboard seeker sensors. As for the term $\dot{\boldsymbol{e}}_{\mathrm{Rate} 0}=\left[\ddot{\theta}_{L 0}, \ddot{\phi}_{L 0}\right]^{T}$, it can be estimated via sampled LOS angles $\theta_{L}, \phi_{L}$ and filter algorithms with recently developed high sampling rate sensors and low-cost computational elements [35]. Such information has been used in the angular acceleration guidance (AAG) law [35] design and implementation. Another alternative is to directly estimate $\dot{\boldsymbol{s}}_{v r 0}$ using $\boldsymbol{s}_{v r}$. Nonetheless, this is not the focus of this work and will not be discussed in detail.

Substituting Eq. (34) into Eq. (32) yields

$$
\dot{s}_{v r}=\varepsilon_{\mathrm{INDI}-\mathrm{TVSMC}}=\Delta D_{r}+\rho(x)
$$

where $\boldsymbol{\varepsilon}_{\text {INDI-TVSMC }}$ is the lumped perturbation. Because of the limited system in practice, $\Delta \boldsymbol{D}_{r}$ is bounded (its upper bound is denoted as $\eta_{1}$ ) during a sampling interval. Moreover, it can be known from Eq. (33) and Assumption 3 that $\rho(x)$ is bounded (its upper bound is denoted as $\eta_{2}$ ) because $\|\Delta \boldsymbol{x}\| \rightarrow 0$ with a sufficiently small $\Delta t$.

Obviously, it can be obtained from Eq. (35) that

$$
\left\|\boldsymbol{\varepsilon}_{\mathrm{INDI}-\mathrm{TVSMC}}\right\| \leq \eta_{1}+\eta_{2}
$$

which means that the perturbation is bounded. To compensate $\boldsymbol{\varepsilon}_{\text {INDI-TVSMC }}$, the incremental TVSMC guidance law is designed as

$$
\Delta \boldsymbol{U}_{\mathrm{INDI}-\mathrm{TVSMC}}=\Delta \boldsymbol{U}_{\mathrm{INDI}-\mathrm{TVSMC}}^{\mathrm{eq}}+\tilde{\boldsymbol{B}}_{0}^{-1} \boldsymbol{v}_{v r}
$$

where

$$
\boldsymbol{v}_{v r}=-\beta_{v r} \frac{\boldsymbol{s}_{v r}}{\left\|\boldsymbol{s}_{v r}\right\|}
$$

with a positive parameter $\beta_{v r}$. Substituting Eq. (37) into Eq. (32) leads to

$$
\dot{\boldsymbol{s}}_{v r}=\boldsymbol{v}_{v r}+\boldsymbol{\varepsilon}_{\mathrm{INDI}-\mathrm{TVSMC}}
$$

Select a Lyapunov function as $W_{v r}=1 / 2 \boldsymbol{s}_{v r}^{T} \boldsymbol{s}_{v r}$, it can be derived that

$$
\begin{aligned}
\dot{W}_{v r} & =\boldsymbol{s}_{v r}^{T}\left(\boldsymbol{v}_{v r}+\boldsymbol{\varepsilon}_{\text {INDI-TVSMC }}\right) \\
& =\boldsymbol{s}_{v r}^{T}\left(-\beta_{v r} \frac{\boldsymbol{s}_{v r}}{\left\|\boldsymbol{s}_{v r}\right\|}+\boldsymbol{\varepsilon}_{\text {INDI-TVSMC }}\right) \\
& \leq-\left(\beta_{v r}-\left\|\boldsymbol{\varepsilon}_{\text {INDI-TVSMC }}\right\|\right)\left\|\boldsymbol{s}_{v r}\right\|
\end{aligned}
$$

To ensure $\dot{W}_{v r}<0$, it can be obtained that

$$
\beta_{v r}>\left\|\varepsilon_{\text {INDI-TVSMC }}\right\|
$$

In other words, $\boldsymbol{s}_{v r}$ can converge to the origin by selecting $\beta_{v r}$ as Eq. (41). After $\boldsymbol{s}_{v r}=0$ is met, the LOS tracking error dynamics has the same form of Eq. (29), which guarantees the convergence of $e_{1}, e_{2}$ to the origin. 
The whole INDI-TVSMC guidance law can be presented as

$$
\boldsymbol{U}_{\text {INDI-TVSMC }}=\boldsymbol{U}_{0}+\Delta \boldsymbol{U}_{\text {INDI-TVSMC }}
$$

where $\boldsymbol{U}_{0}=\left[A_{z M 0}, A_{y M 0}\right]^{T}$ denotes the missile's guidance command at $t_{0}$.

Remark 5: It is worth noting that the proposed INDI guidance law differs from the LOS angular acceleration guidance law [35] in that 1) both the LOS angular acceleration and guidance command at their latest step are used in the INDI guidance law, and 2) the guidance robustness and performance are enhanced by inheriting the advantages of both INDI and SMC/TVSMC controls.

Similar to the design process of the INDI-TVSMC guidance law, the whole INDI-SMC guidance law can be presented as

$$
\boldsymbol{U}_{\mathrm{INDI}-\mathrm{SMC}}=\boldsymbol{U}_{0}+\Delta \boldsymbol{U}_{\mathrm{INDI}-\mathrm{SMC}}
$$

where

$$
\begin{gathered}
\Delta \boldsymbol{U}_{\mathrm{INDI}-\mathrm{SMC}}=\Delta \boldsymbol{U}_{\mathrm{INDI}-\mathrm{SMC}}^{\mathrm{eq}}+\tilde{\boldsymbol{B}}_{0}^{-1} \boldsymbol{v}_{r} \\
\Delta \boldsymbol{U}_{\mathrm{INDI}-\mathrm{SMC}}^{\mathrm{eq}}=-\tilde{\boldsymbol{B}}_{0}^{-1} \dot{\boldsymbol{s}}_{r 0} \\
\boldsymbol{v}_{r}=-\beta_{r} \frac{\boldsymbol{s}_{r}}{\left\|\boldsymbol{s}_{r}\right\|}
\end{gathered}
$$

with $\beta_{r}>0, \boldsymbol{s}_{r}$ has the same form of $\boldsymbol{s}$ in Eq. (12), and $\dot{\boldsymbol{s}}_{r 0}=r_{0} \dot{\boldsymbol{e}}_{\text {Rate } 0}+\left(\dot{r}_{0}+k\right) \boldsymbol{e}_{\text {Rate } 0}$ can be similarly estimated/measured as claimed in Remark 4. Similar to Eq. (35), $\boldsymbol{\varepsilon}_{\text {INDI-SMC }}$ can be presented as

$$
\boldsymbol{\varepsilon}_{\mathrm{INDI}-\mathrm{SMC}}=\Delta \boldsymbol{D}_{r}+\boldsymbol{\rho}^{\prime}(\boldsymbol{x})
$$

where

$$
\rho^{\prime}(\boldsymbol{x})=\left.\frac{\partial[\tilde{\boldsymbol{A}}(\boldsymbol{x})+\tilde{\boldsymbol{B}}(\boldsymbol{x}) \boldsymbol{U}]}{\partial \boldsymbol{x}}\right|_{0} \Delta \boldsymbol{x}+\mathcal{O}\left(\Delta \boldsymbol{x}^{2}\right)
$$

Similarly, the system perturbation $\boldsymbol{\varepsilon}_{\mathrm{INDI}-\mathrm{SMC}}$ is bounded by

$$
\left\|\boldsymbol{\varepsilon}_{\mathrm{INDI}-\mathrm{SMC}}\right\| \leq \eta_{1}+\eta_{3}
$$

where $\eta_{3}$ is the upper bound of $\boldsymbol{\rho}^{\prime}(\boldsymbol{x})$. Moreover, $\boldsymbol{s}_{r}$ can converge to the origin by selecting $\beta_{r}$ as

$$
\beta_{r}>\left\|\boldsymbol{\varepsilon}_{\mathrm{INDI}-\mathrm{SMC}}\right\|
$$

After $\boldsymbol{s}_{r}=0$ is met, the LOS tracking error dynamics has the same form of Eq. (20), which guarantees the convergence of $e_{1}, e_{2}$ to the origin.
The structure of the NDI-SMC-based, NDI-TVSMC-based, INDISMC-based, and INDI-TVSMC-based guidance laws can be presented in Fig. 5. Therein, four switch points (denoted as SP1, SP2, SP3, and SP4) are used to trigger different guidance laws. Specifically, if SP1 and SP2 are connected to the "TVSMC" block, the INDITVSMC-based or NDI-TVSMC-based guidance law is used when SP3 and SP4 connect to the solid line or dashed lines, respectively. If SP1 and SP2 are connected to the "SMC" block, the INDI-SMCbased or NDI-SMC-based guidance law is used when SP3 and SP4 connect to the solid line or dashed lines, respectively. It can be seen that the INDI-based TVSMC and SMC guidance laws exploit the sensor-based estimation/measurement of $\dot{\boldsymbol{s}}_{v r 0}$ or $\dot{\boldsymbol{s}}_{r 0}$, whereas the NDI-based TVSMC and SMC guidance laws use the information $\tilde{\boldsymbol{A}}_{v}$ or $\tilde{\boldsymbol{A}}$.

\section{E. Robustness Comparisons}

To compare the above guidance laws for practical application, the NDI-SMC-based guidance law is first analyzed by applying it into the uncertain system as follows:

$$
\dot{\boldsymbol{s}}=\tilde{\boldsymbol{A}}+\tilde{\boldsymbol{B}} \boldsymbol{U}+\boldsymbol{D}_{r}
$$

where $\boldsymbol{D}_{r}$ is presented in Eq. (31). Substituting $\boldsymbol{U}_{\mathrm{NDI}-\mathrm{SMC}}$ [Eq. (16)] into Eq. (51) leads to

$$
\dot{\boldsymbol{s}}=\boldsymbol{v}+\boldsymbol{\varepsilon}_{\mathrm{NDI}-\mathrm{SMC}}
$$

where $\boldsymbol{\varepsilon}_{\mathrm{NDI}-\mathrm{SMC}}=\boldsymbol{D}_{r}$. For the Lyapunov function $W_{1}=1 / 2 \boldsymbol{s}^{T} \boldsymbol{s}$, it can be derived that

$$
\begin{aligned}
\dot{W}_{1} & =s^{T}\left(v+\varepsilon_{\mathrm{NDI}-\mathrm{SMC}}\right)=\boldsymbol{s}^{T}\left(-\beta \frac{\boldsymbol{s}}{\|\boldsymbol{s}\|}+\boldsymbol{\varepsilon}_{\mathrm{NDI}-\mathrm{SMC}}\right) \\
& \leq-\left(\beta-\left\|\boldsymbol{\varepsilon}_{\mathrm{NDI}-\mathrm{SMC}}\right\|\right)\|\boldsymbol{s}\|
\end{aligned}
$$

Because the perturbation $\boldsymbol{\varepsilon}_{\mathrm{NDI}-\mathrm{SMC}}$ is bounded, the convergence of $\boldsymbol{s}$ can be ensured by the condition $\beta>\left\|\boldsymbol{\varepsilon}_{\mathrm{NDI}-\mathrm{SMC}}\right\|$. For the NDITVSMC-based guidance law in Eq. (27), the system perturbation is $\boldsymbol{\varepsilon}_{\mathrm{NDI}-\mathrm{TVSMC}}=\boldsymbol{D}_{r}=\boldsymbol{\varepsilon}_{\mathrm{NDI}-\mathrm{SMC}}$. To ensure the convergence of $\boldsymbol{s}_{v}, \beta_{v}$ should meet $\beta_{v}>\left\|\boldsymbol{\varepsilon}_{\mathrm{NDI}-\mathrm{TVSMC}}\right\|$.

From the analysis results in Sec. III.D, the perturbations resulted from different guidance laws under the uncertain system are compared in Table 1 . The parameter selections for perturbation cancellation of each guidance law are also presented. Because the parameter selections are related to the norm of the perturbations, the following relations are presented:

$$
\begin{gathered}
\left\|\boldsymbol{\varepsilon}_{\mathrm{INDI}-\mathrm{TVSMC}}\right\|=\left\|\Delta \boldsymbol{D}_{r}+\boldsymbol{\rho}\right\| \leq\left\|\Delta \boldsymbol{D}_{r}\right\|+\|\boldsymbol{\rho}\| \\
\left\|\boldsymbol{\varepsilon}_{\mathrm{INDI}-\mathrm{SMC}}\right\|=\left\|\Delta \boldsymbol{D}_{r}+\boldsymbol{\rho}^{\prime}\right\| \leq\left\|\Delta \boldsymbol{D}_{r}\right\|+\left\|\boldsymbol{\rho}^{\prime}\right\|
\end{gathered}
$$

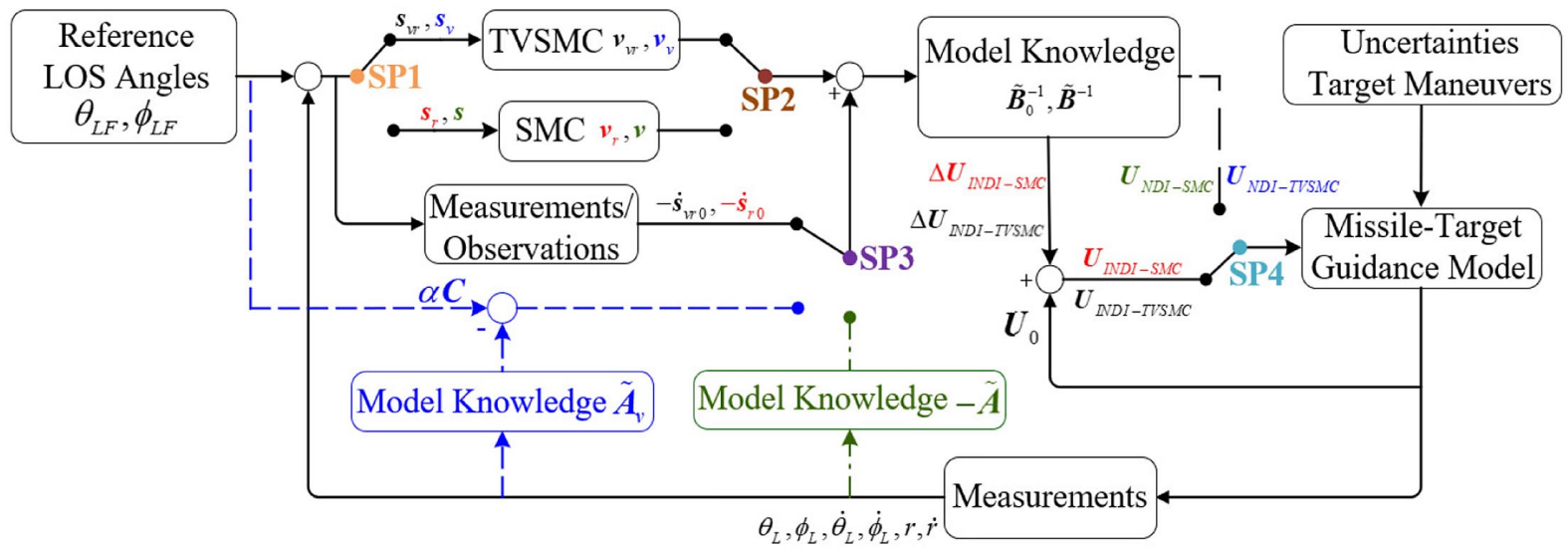

Fig. 5 Structure of the NDI-SMC-based, NDI-TVSMC-based, INDI-SMC-based, and INDI-TVSMC-based guidance laws. 
Table 1 Comparison of perturbations and conditions for different guidance laws

\begin{tabular}{lcc}
\hline \hline Guidance law & Perturbations in the uncertain system & Parameter selection \\
\hline INDI-TVSMC & $\boldsymbol{\varepsilon}_{\text {INDI-TVSMC }}=\Delta \boldsymbol{D}_{r}+\boldsymbol{\rho}$ & $\beta_{v r}>\left\|\boldsymbol{\varepsilon}_{\text {INDI-TVMCC }}\right\|$ \\
INDI-SMC & $\boldsymbol{\varepsilon}_{\text {INDI-SMC }}=\Delta \boldsymbol{D}_{r}+\boldsymbol{\rho} \prime$ & $\beta_{r}>\left\|\boldsymbol{\varepsilon}_{\text {INDI-SMC }}\right\|$ \\
NDI-TVSMC & $\boldsymbol{\varepsilon}_{\text {NDI-TVSMC }}=\boldsymbol{D}_{r}$ & $\beta_{v}>\left\|\boldsymbol{\varepsilon}_{\text {NDI-TVSMC }}\right\|$ \\
NDI-SMC & $\boldsymbol{\varepsilon}_{\text {NDI-SMC }}=\boldsymbol{D}_{r}$ & $\beta>\left\|\boldsymbol{\varepsilon}_{\text {NDI-SMC }}\right\|$ \\
\hline \hline
\end{tabular}

$$
\begin{gathered}
\left\|\boldsymbol{\varepsilon}_{\mathrm{NDI}-\mathrm{TVSMC}}\right\|=\left\|\boldsymbol{D}_{r}\right\| \\
\left\|\boldsymbol{\varepsilon}_{\mathrm{NDI}-\mathrm{SMC}}\right\|=\left\|\boldsymbol{D}_{r}\right\|
\end{gathered}
$$

According to Eqs. (33) and (48), $\|\boldsymbol{\rho}\|$ and $\| \boldsymbol{\rho}$ ' $\|$ can be negligible because $\|\Delta \boldsymbol{x}\| \rightarrow 0$ with a sufficiently small $\Delta t$. Meanwhile, if $\boldsymbol{D}_{r} \neq 0$, there exists a sufficiently small $\Delta t$ such that $\left\|\Delta \boldsymbol{D}_{r}\right\|<\left\|\boldsymbol{D}_{r}\right\|[32,33]$. This implies that a sufficiently small $\Delta t$ can guarantee that the following conditions are satisfied:

$$
\begin{aligned}
\left\|\boldsymbol{\varepsilon}_{\mathrm{INDI}-\mathrm{TVSMC}}\right\| & <\left\|\boldsymbol{\varepsilon}_{\mathrm{NDI}-\mathrm{TVSMC}}\right\|, & & \left\|\boldsymbol{\varepsilon}_{\mathrm{INDI}-\mathrm{TVSMC}}\right\|<\left\|\boldsymbol{\varepsilon}_{\mathrm{NDI}-\mathrm{SMC}}\right\|, \\
\left\|\boldsymbol{\varepsilon}_{\mathrm{INDI}-\mathrm{SMC}}\right\| & <\left\|\boldsymbol{\varepsilon}_{\mathrm{NDI}-\mathrm{SMC}}\right\|, & & \left\|\boldsymbol{\varepsilon}_{\mathrm{INDI}-\mathrm{SMC}}\right\|<\left\|\boldsymbol{\varepsilon}_{\mathrm{NDI}-\mathrm{TVSMC}}\right\|
\end{aligned}
$$

This means that $\beta_{v r}$ and $\beta_{r}$ can be selected smaller than $\beta_{v}$ and $\beta$ under the same system uncertainties and target maneuvers.

Remark 6: The gains $\beta_{v r}, \beta_{r}, \beta_{v}, \beta$ are required to be greater than the corresponding perturbations as shown in Table 1. It is alternative to update $\beta_{v r}, \beta_{r}, \beta_{v}, \beta$ via the adaptive sliding mode control [24,29]. However, it is not the focus in this work and does not affect the discussion of perturbations under the above guidance laws.

\section{Numerical Simulations}

In this section, the NDI-SMC-based, INDI-SMC-based, NDITVSMC-based, INDI-TVSMC-based guidance laws are validated via numerical simulations against constant moving targets and maneuvering targets with desired terminal LOS angles. A realistic missile model $[36,37]$ is considered for verification. The speed and thrust $T_{m}$ are governed by Eqs. (59) and (60) in this work. $D_{m}=$ $0.5 \bar{\rho} V_{M}^{2} S_{\text {ref }} C_{D}$ is the aerodynamic drag, where $C_{D}, \bar{\rho}$ denote the drag coefficient and atmospheric density calculated from [36], $g=9.81 \mathrm{~m} / \mathrm{s}^{2}$ is the gravity constant, and $S_{\text {ref }}=0.1 \mathrm{~m}^{2}$ and $M_{m}=80 \mathrm{~kg}$ are selected for the missile's reference area and mass, respectively.

$$
\dot{V}_{M}=\left(T_{m}-D_{m}\right) / M_{m}-g\left(\cos \phi_{M} \cos \theta_{M} \sin \theta_{L}+\sin \theta_{M} \cos \theta_{L}\right)
$$

$$
T_{m}= \begin{cases}1000 \mathrm{~N}, & \text { for } 0 \leq t \leq 3 \mathrm{~s} \\ 750 \mathrm{~N}, & \text { for } 3<t \leq 10 \mathrm{~s} \\ 0, & \text { for } t>10 \mathrm{~s}\end{cases}
$$

The initial positions are set to $\left(x_{M 0}, y_{M 0}, z_{M 0}\right)=(0,0,10) \mathrm{km}$ and $\left(x_{T 0}, y_{T 0}, z_{T 0}\right)=(10,10,0) \mathrm{km}$, and the initial flight path angles are $\gamma_{M 0}=\vartheta_{M 0}=30^{\circ}, \gamma_{T 0}=\vartheta_{T 0}=0^{\circ}$. The initial speeds of the missile and target are 500 and $200 \mathrm{~m} / \mathrm{s}$, respectively. Both the missile accelerations $A_{z M}$ and $A_{y M}$ are bounded by $200 \mathrm{~m} / \mathrm{s}^{2}$. For all the cases, if not given specifically, the parameters of the above guidance laws are selected as listed in Table 2. Therein, the parameters for NDI-SMC and INDI-SMC, NDI-TVSMC, and INDITVSMC are the same for fair comparison. Simulations are terminated when the relative range is less than $1 \mathrm{~m}$ or the missile misses the target. The unit vector $S /\|S\|$ ( $S$ denotes the sliding variable under each guidance law) is modified as $\boldsymbol{S} /(\|S\|+0.01)$ to avoid undesirable chattering.
Table 2 Guidance parameters for different guidance laws

\begin{tabular}{lc}
\hline \hline Guidance law & Guidance parameters \\
\hline INDI-TVSMC & $k_{v 0}=100, \alpha=50, \beta_{v r}=15$ \\
INDI-SMC & $k=700, \beta_{r}=15$ \\
NDI-TVSMC & $k_{v 0}=100, \alpha=50, \beta_{v}=15$ \\
NDI-SMC & $k=700, \beta=15$ \\
\hline \hline
\end{tabular}

\section{A. Constant Moving Targets Under Nominal Conditions}

The first set of simulations is to verify the feasibility of the proposed guidance laws against constant moving targets under nominal conditions. If not specifically emphasized, the thin lines represent the state variations in the pitch plane in the upcoming figures. The simulation results under $\theta_{L F}=-30^{\circ}, \phi_{L F}=30^{\circ}$ are presented in Figs. 6 and 7. It can be seen that all the guidance laws successfully intercept the target via various trajectories created by different guidance laws. In Fig. 7, it can be observed that the LOS angles and LOS rates driven by the INDI-TVSMC and NDI-TVSMC guidance laws converge to their desired values faster than that by INDI-SMC and NDI-SMC guidance laws. All the guidance laws can generate feasible and reasonable acceleration commands, and all the LOS angle tracking errors converge to zero before collision.

Besides, another pair of the constraint $\theta_{L F}=0^{\circ}, \phi_{L F}=0^{\circ}$ is selected to verify the guidance performance. In Figs. 8 and 9, the $3 \mathrm{D}$ trajectories and guidance performance are obtained using the same parameters listed in Table 2. The INDI-TVSMC and NDITVSMC guidance laws generate greater guidance commands than the NDI-SMC and INDI-SMC guidance laws to eliminate the LOS tracking errors. It can be seen that the INDI-TVSMC and NDITVSMC guidance laws can achieve the desired LOS angle constraint, whereas the NDI-SMC and INDI-SMC guidance laws cannot. This is because the initial sliding variables for NDI-SMC and INDI-SMC become too large to be eliminated by the gains listed in Table 2 . To handle this issue, there are two ways for NDI-SMC and INDI-SMC guidance laws: 1 ) increase $\beta_{r}$ and $\beta$ to accelerate convergence of the sliding variables; 2) select smaller $k$ to reduce the initial sliding variable such that the sliding variable can be driven to zero before collision. The results are shown in Fig. 10. It can be seen that the LOS tracking errors can converge to zero by increasing $\beta_{r}$ and $\beta$ to 30, and reach zero on collision by decreasing $k$ to 200 . However, these high gains of $\beta_{r}$ and $\beta$ lead to undesirable chattering in the guidance commands. Because the LOS tracking errors under $k=200$ are still nonzero near collision, the guidance commands diverge because $\dot{\theta}_{L}=\dot{e}_{1}=-(k / r) e_{1} \rightarrow \infty$ and $\dot{\phi}_{L}=\dot{e}_{2}=-(k / r) e_{2} \rightarrow \infty$ as $r \rightarrow 0$. These disadvantages are consistent with the discussions in Remark 3. It can be concluded that the NDI-TVSMC and

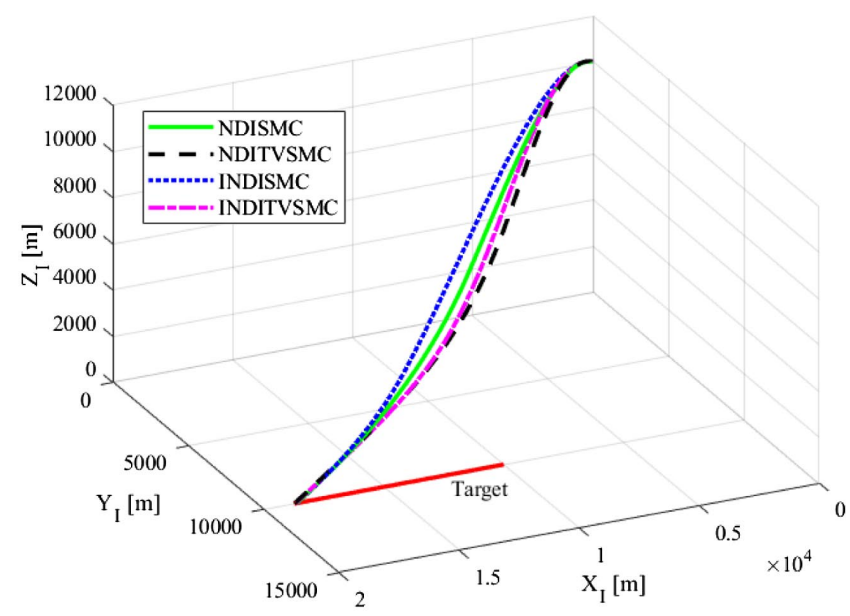

Fig. 6 Three-dimensional trajectories against the constant moving target under $\theta_{L F}=-30^{\circ}, \phi_{L F}=30^{\circ}$. 

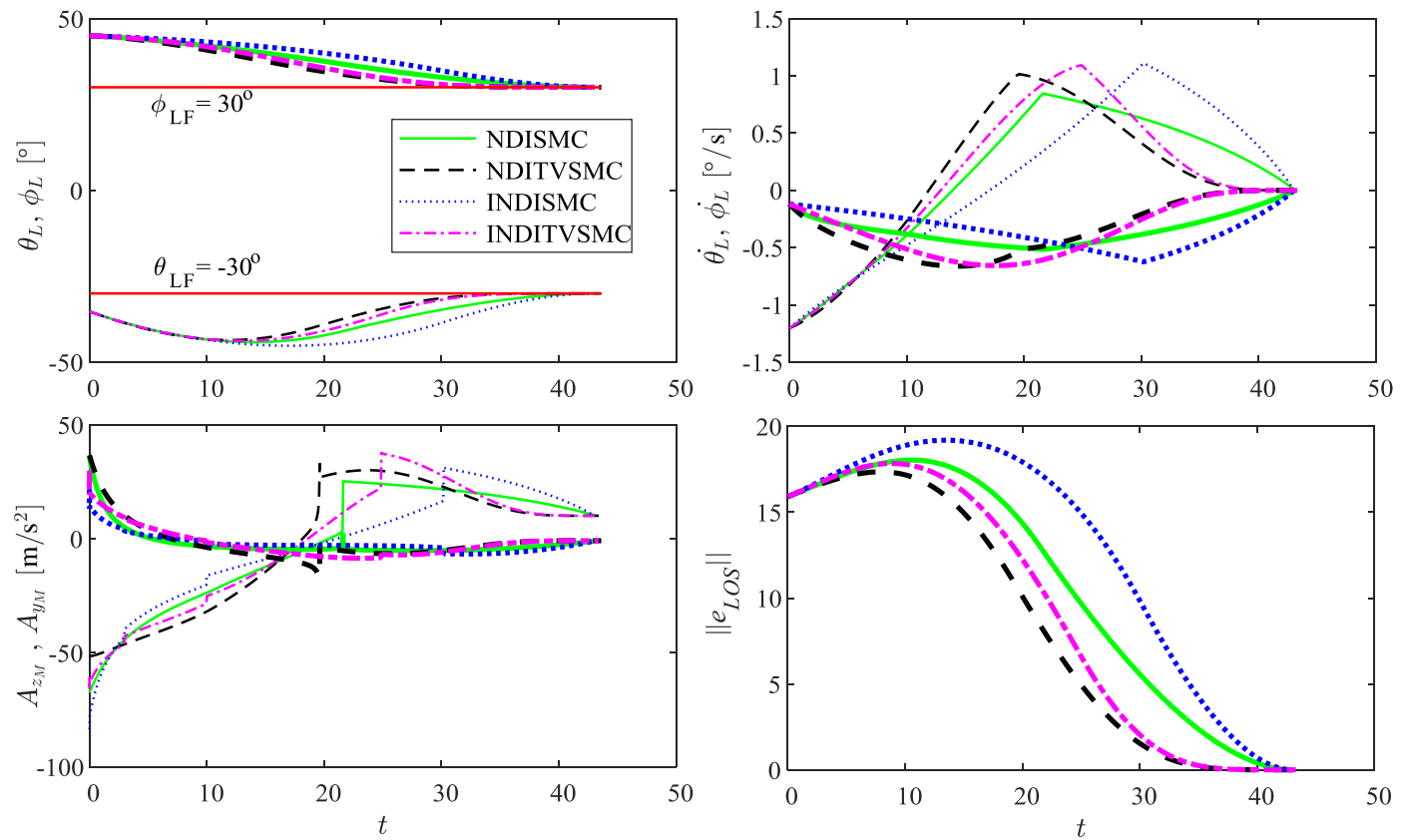

Fig. 7 Guidance performance against the constant moving target under $\theta_{L F}=-30^{\circ}, \phi_{L F}=30^{\circ}$.

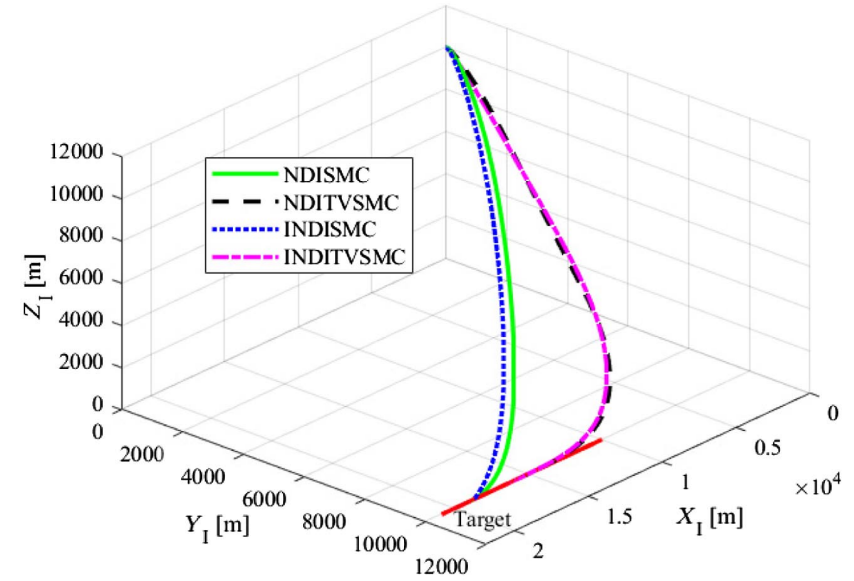

Fig. 8 Three-dimensional trajectories against the constant moving target under $\theta_{L F}=0^{\circ}, \phi_{L F}=0^{\circ}$.
INDI-TVSMC guidance laws are more feasible than the NDI-SMC and INDI-SMC guidance laws.

\section{B. Maneuvering Targets Under Nominal Conditions}

To validate the guidance law against maneuvering targets, the target acceleration is assumed to have the form of $A_{z T}=a_{z} \cos (\varpi t)$ and $A_{y T}=a_{y} \sin (\varpi t)$. The constraint $\theta_{L F}=-30^{\circ}, \phi_{L F}=30^{\circ}$ is selected. Firstly, the results under $a_{z}=a_{y}=5, \varpi=0.3$ are presented in Figs. 11 and 12. It can be seen that the target is successfully intercepted by all the guidance laws. Again, it can be observed that the LOS angles can converge to their desired values by the INDI-TVSMC and NDI-TVSMC guidance laws faster than that by the INDI-SMC and NDI-SMC guidance laws. Note that all the guidance laws can create feasible guidance commands with bounded terminal acceleration even in the case of unknown target maneuvers.

Meanwhile, the guidance laws are verified by increasing the target maneuvers to $a_{z}=a_{y}=30$. The results are shown in Figs. 13 and 14. Note that all the guidance laws can ensure the successful interception of the target. The INDI-SMC and INDI-TVSMC guidance

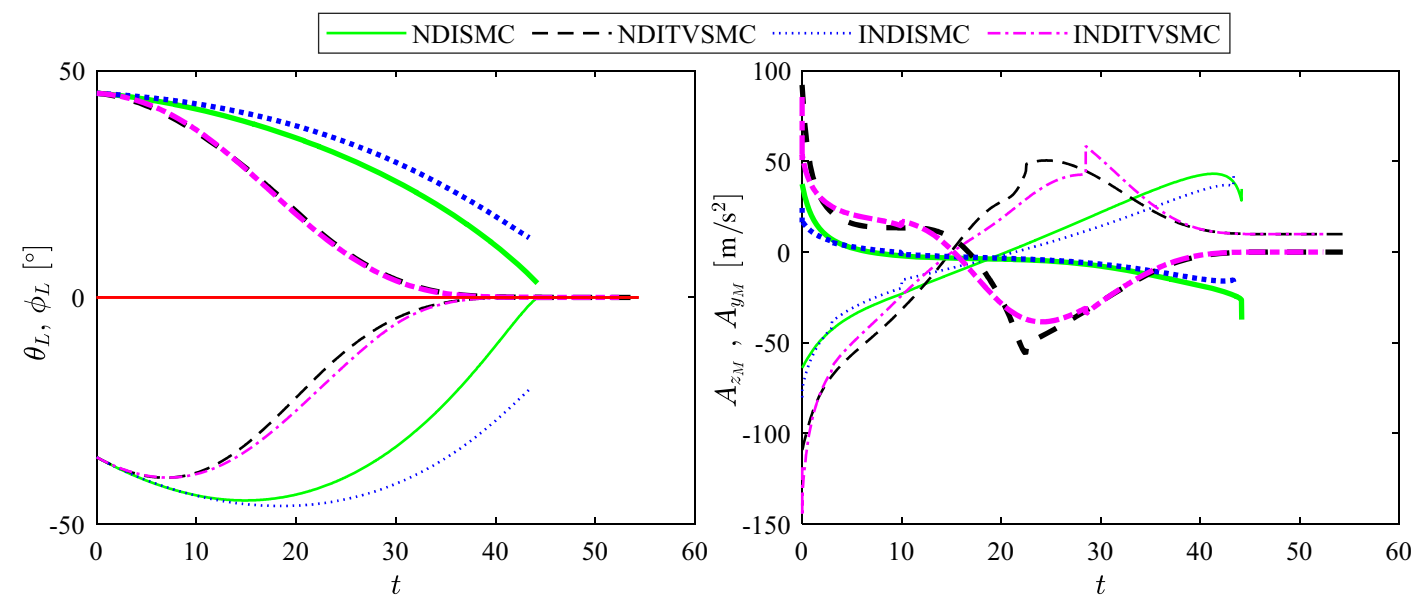

Fig. 9 Guidance performance against the constant moving target under $\theta_{L F}=0^{\circ}, \phi_{L F}=0^{\circ}$. 

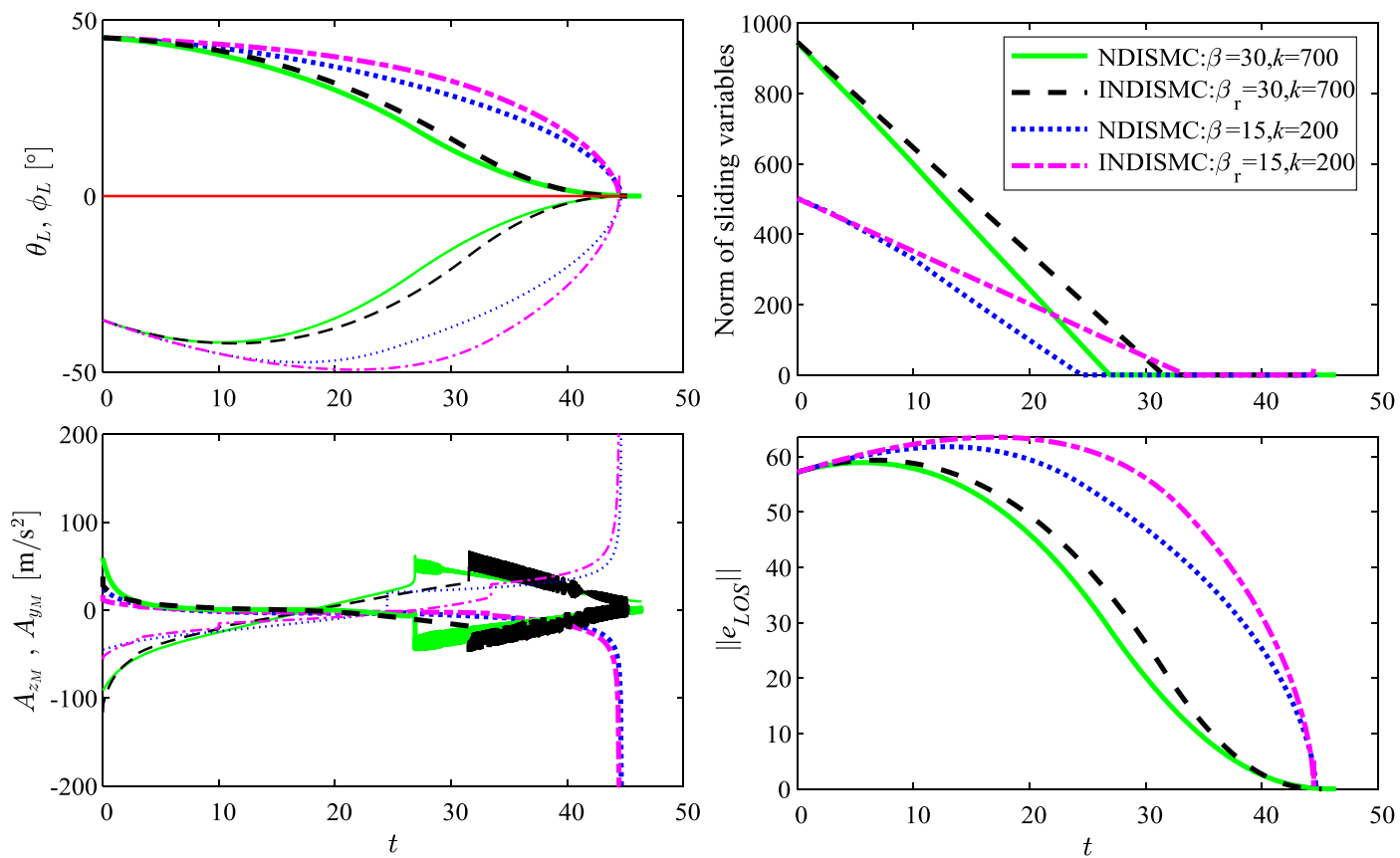

Fig. 10 Gains tuning for NDI-SMC and INDI-SMC against the constant moving target under $\theta_{L F}=0^{\circ}, \phi_{L F}=0^{\circ}$.

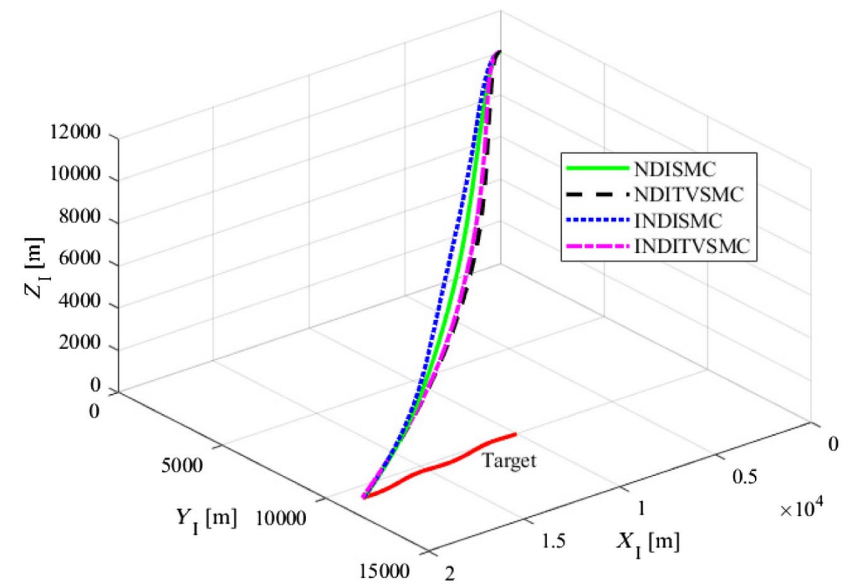

Fig. 11 Three-dimensional trajectories against the maneuvering target under $A_{z T}=5 \cos (0.3 t), A_{y T}=5 \sin (0.3 t)$.

laws can enforce the LOS tracking errors to zero before interception using the same gains listed in Table 2. However, the NDI-SMC and NDI-TVSMC guidance laws cannot achieve the desired LOS angles because the increased target maneuvers lead to higher perturbations

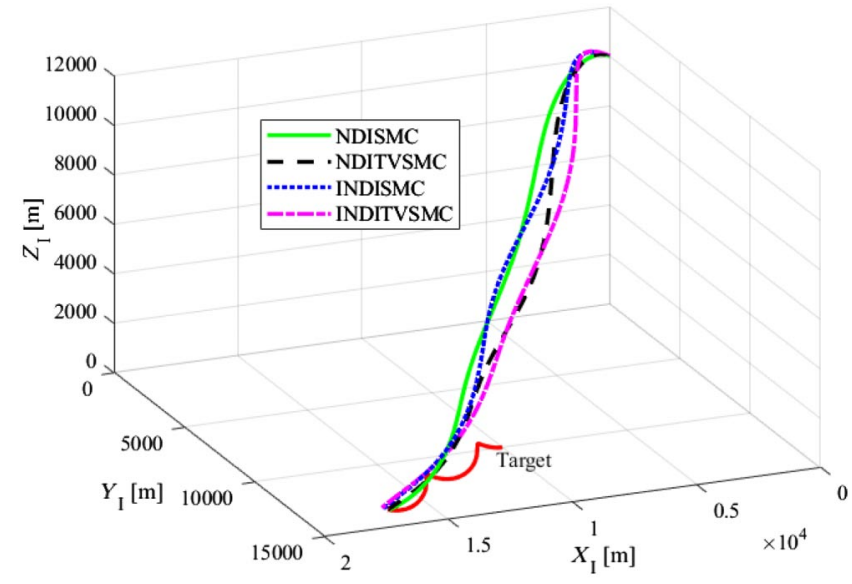

Fig. 13 Three-dimensional trajectories against the maneuvering target under $A_{z T}=30 \cos (0.3 t), A_{y T}=30 \sin (0.3 t)$.

that are greater than the gains listed in Table 2 . By equally increasing the gains $\beta_{v}$ and $\beta$ to 30 and 45 , the results for the NDI-SMC and NDITVSMC guidance laws are presented in Fig. 15. Compared with the results by NDI-SMC and NDI-TVSMC guidance laws in Fig. 14, the
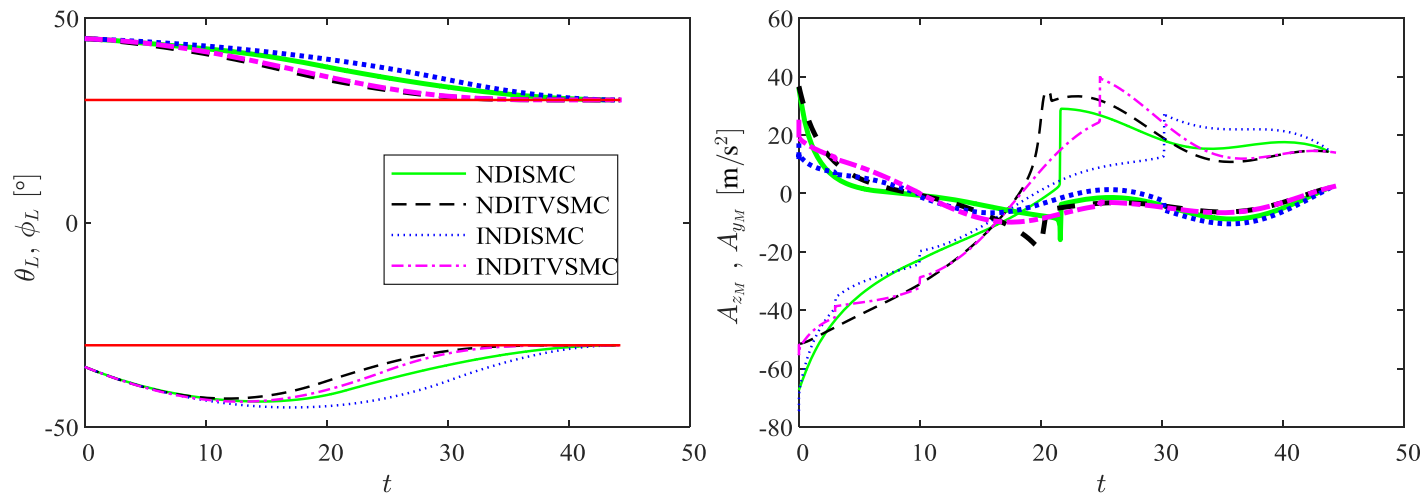

Fig. 12 Guidance performance against the maneuvering target under $A_{z T}=5 \cos (0.3 t), A_{y T}=5 \sin (0.3 t)$. 

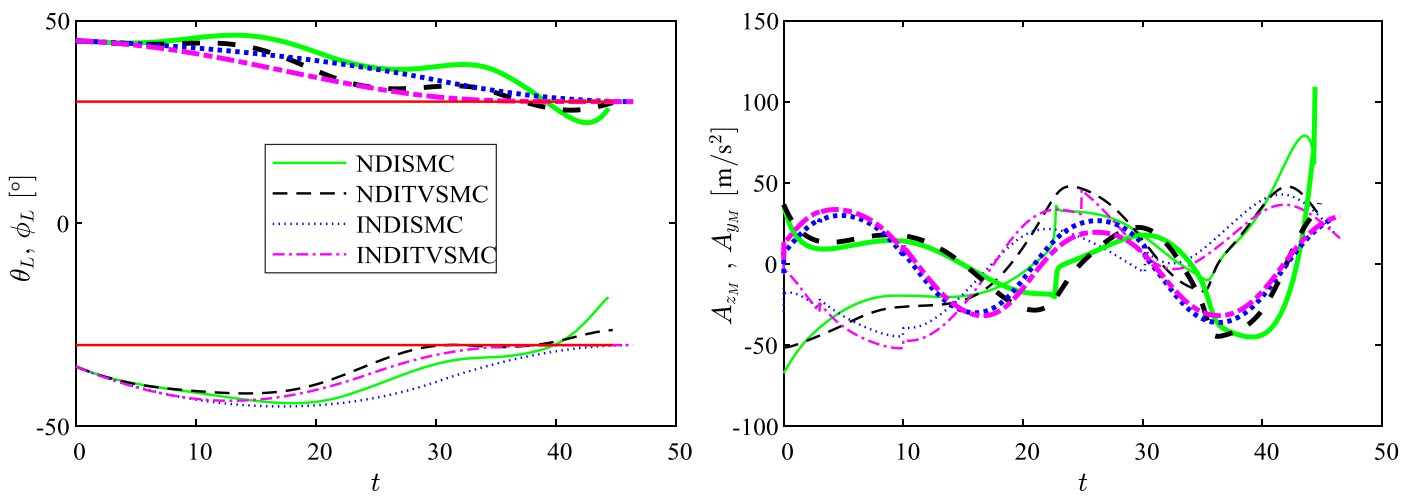

Fig. 14 Guidance performance against the maneuvering target under $A_{z T}=30 \cos (0.3 t), A_{y T}=30 \sin (0.3 t)$.
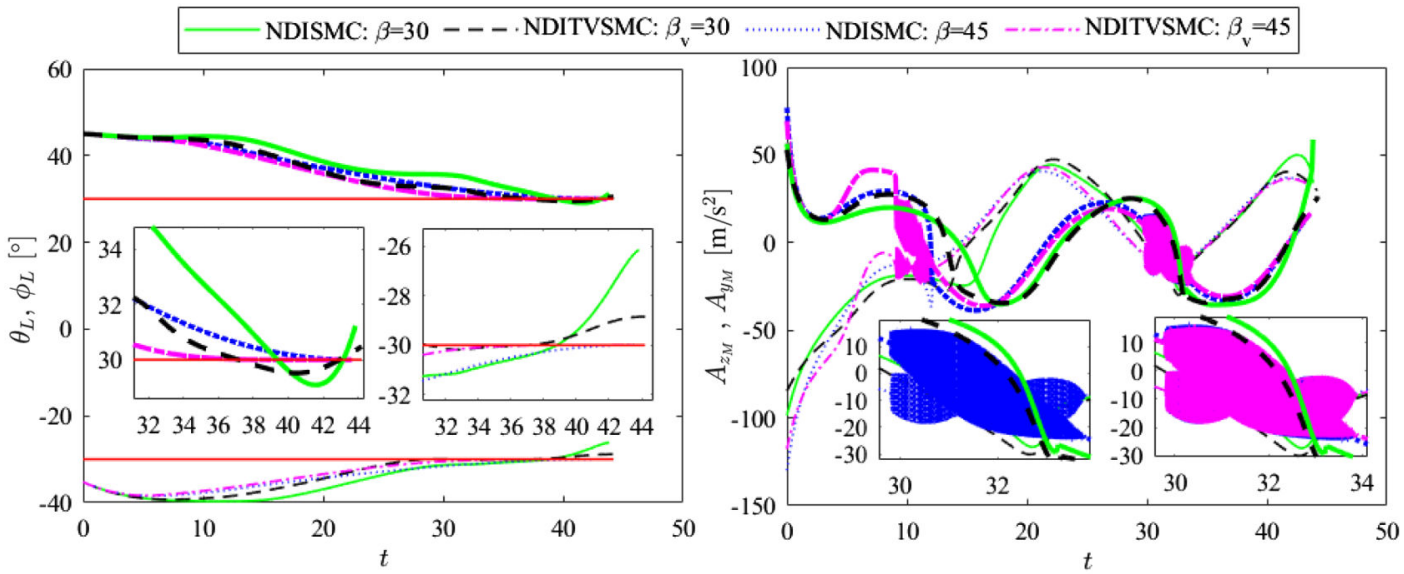

Fig. 15 Results for NDI-SMC and NDI-TVSMC under $A_{z T}=30 \cos (0.3 t), A_{y T}=30 \sin (0.3 t)$ with higher gains.

terminal LOS angle errors become smaller with higher gains of $\beta_{v}, \beta$. Although the desired LOS angles are achieved by $\beta_{v}=\beta=45$, undesirable chattering is induced, as can be seen from Fig. 15 .

To show the guidance feasibility under discontinuous target maneuvers that do not satisfy Assumption 2, the target lateral accelerations are assumed to change abruptly every $15 \mathrm{~s}$ with the profiles shown in Fig. 16. The 3D trajectories and guidance performance are shown in Figs. 17 and 18. All the guidance laws can successfully intercept the target. The missile under INDI-SMC and INDI-TVSMC can meet the expected LOS angles before collision using the gains in Table 2. However, the NDI-SMC and NDI-TVSMC guidance laws cannot achieve the desired LOS angles because the selected gains $\beta=\beta_{v}=15$ cannot cancel the resulting perturbations. By

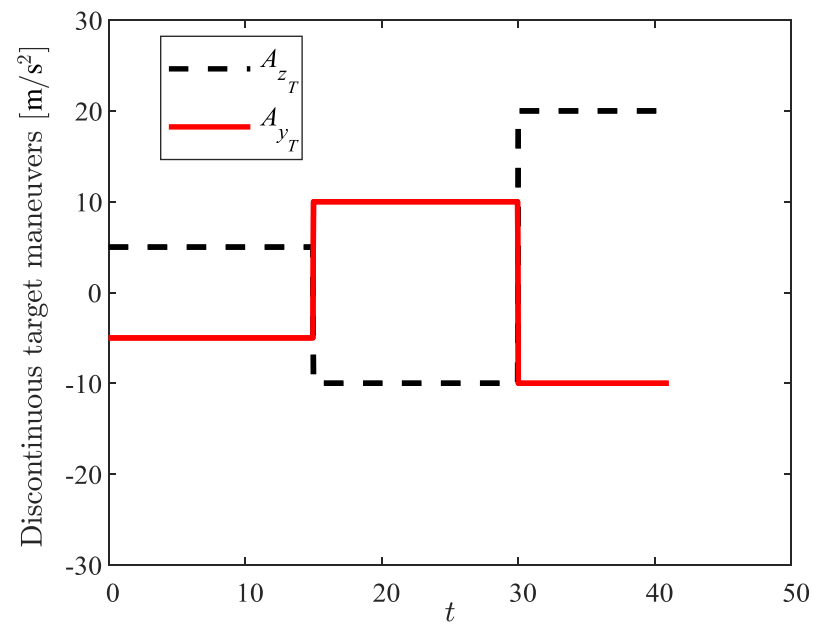

Fig. 16 Discontinuous target maneuver profiles.

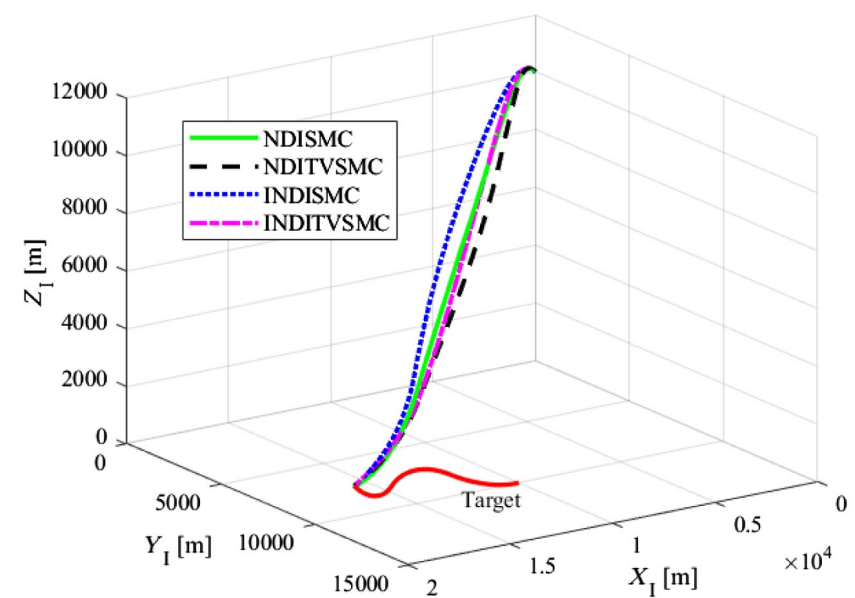

Fig. 17 Three-dimensional trajectories against discontinuous target maneuvers.

increasing the gains $\beta_{v}$ and $\beta$ to 30 , the NDI-SMC and NDI-TVSMC guidance performances are presented in Fig. 19. Compared with the results under NDI-SMC and NDI-TVSMC with $\beta=\beta_{v}=15$, zero LOS tracking errors are achieved. However, similar to the results in Fig. 15, undesirable chattering occurs in guidance commands.

\section{Monte Carlo Simulations Under Uncertain Conditions}

In this subsection, the dispersions in the missile's initial flight path angles and initial positions are assumed to follow normal distributions with zero means, and standard deviations of $1 \mathrm{deg}$ and $500 \mathrm{~m}$, respectively. Moreover, a standard deviation of $10 \mathrm{~N}$ is assumed for the thrust uncertainty, $1 \mathrm{deg}$ for the LOS angle measurement noise, 

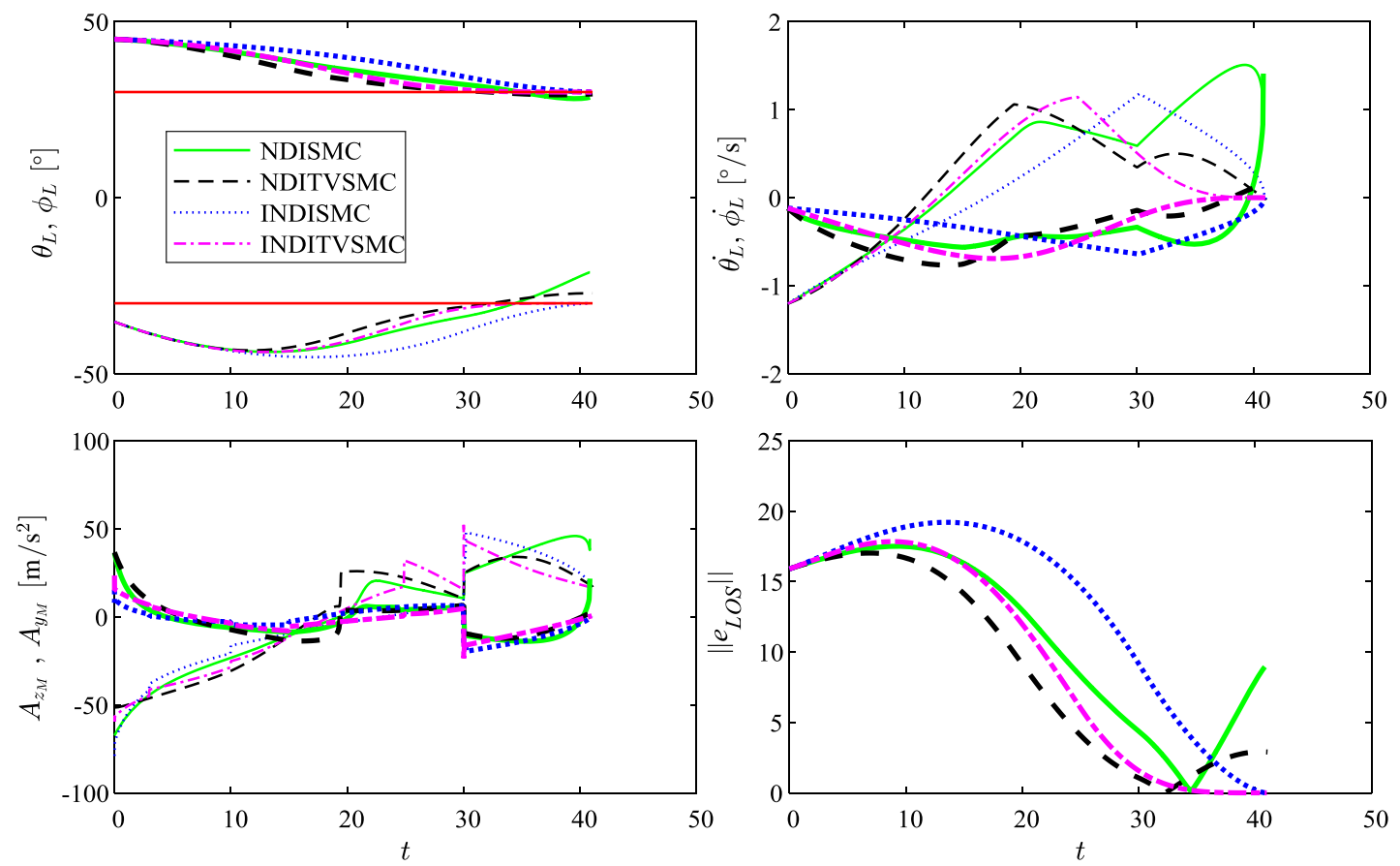

Fig. 18 Guidance performance against discontinuous target maneuvers under $\theta_{L F}=-30^{\circ}, \phi_{L F}=30^{\circ}$.
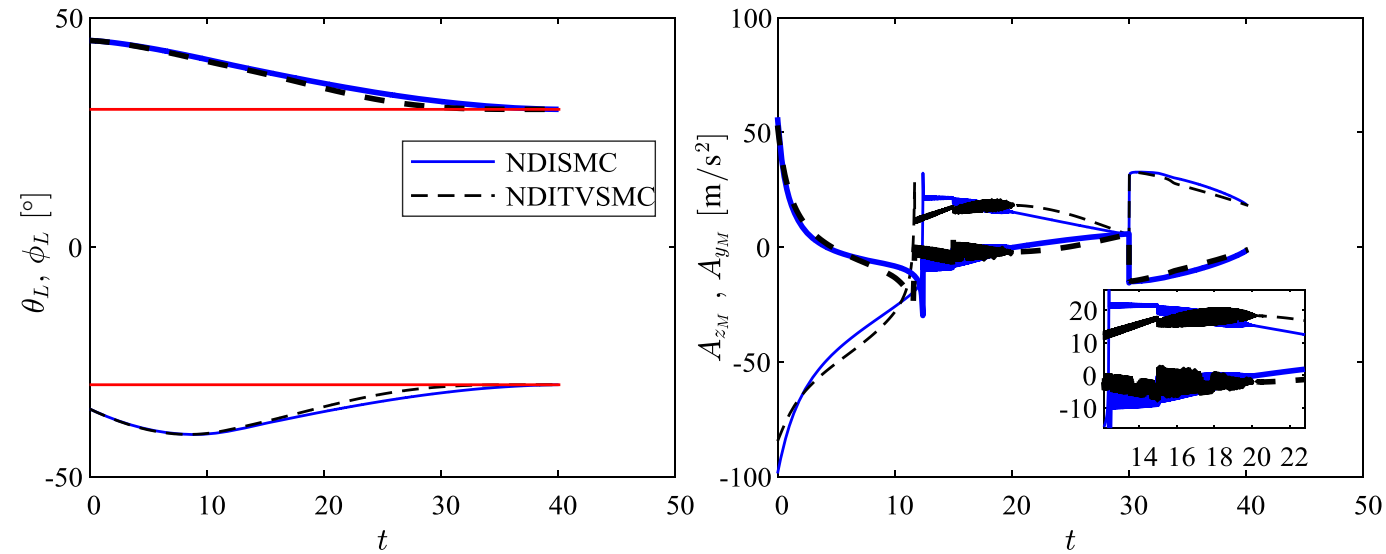

Fig. 19 Results for NDI-SMC and NDI-TVSMC against discontinuous target maneuvers with higher gains.

$10 \mathrm{~m} / \mathrm{s}$ for the relative velocity measurement noise, and $100 \mathrm{~m}$ for the relative range measurement noise. In addition, because the sensorbased guidance methods require the LOS angular acceleration, a standard deviation of $0.02 \mathrm{rad} / \mathrm{s}^{2}$ is assumed for the LOS angular acceleration measurement noise for INDI-SMC and INDI-TVSMC guidance laws.

By employing the above settings and the guidance parameters in Table 2, 500 Monte Carlo runs are performed for each guidance law against the maneuvering target with the constraint $\theta_{L F}=-30^{\circ}$, $\phi_{L F}=30^{\circ}$. The distributions of the miss distance and terminal LOS angle errors under $A_{z T}=5 \cos (0.3 t), A_{y T}=5 \sin (0.3 t)$ are presented in Figs. 20-22. It can be seen from Fig. 20 that all the guidance laws are capable of intercepting the target with the miss distance less than $1 \mathrm{~m}$. In Fig. 21, it can be observed that different distribution results are obtained by different guidance laws. Specifically, more than $90 \%$ of the $\theta_{L}$ errors are smaller than 0.05 deg under
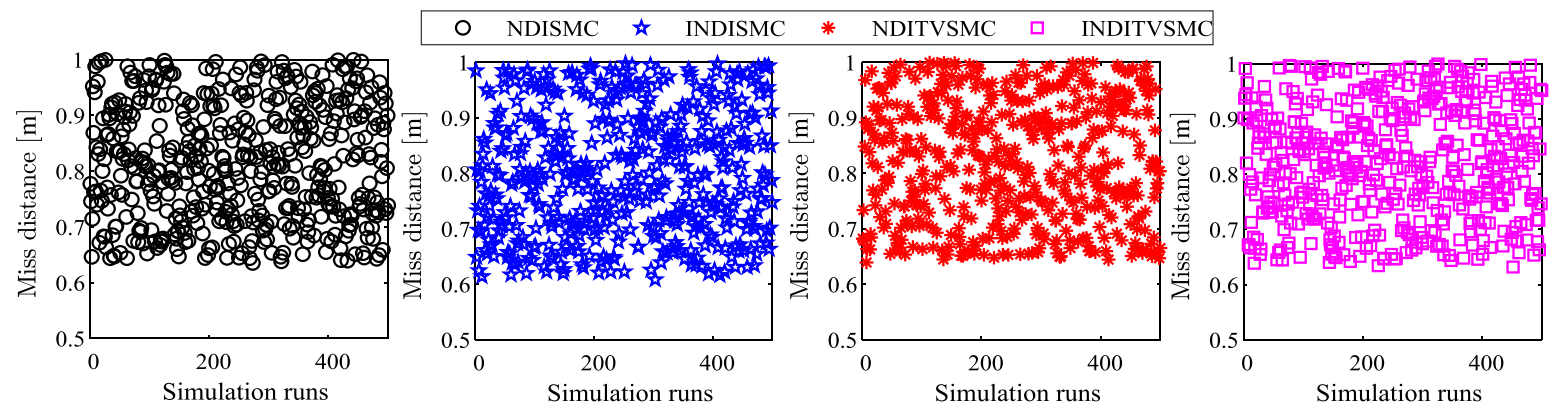

Fig. 20 Miss distance distribution under $A_{z T}=5 \cos (0.3 t), A_{y T}=5 \sin (0.3 t)$ and $\theta_{L F}=-30^{\circ}, \phi_{L F}=30^{\circ}$. 


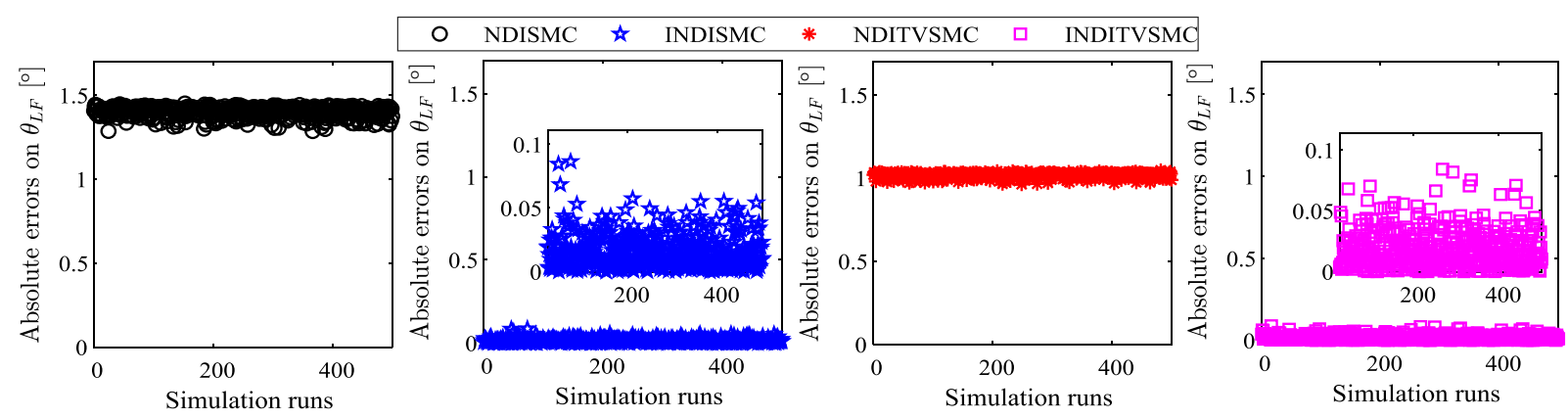

Fig. 21 Distribution of $\theta_{L}$ errors under $A_{z T}=5 \cos (0.3 t), A_{y T}=5 \sin (0.3 t)$ and $\theta_{L F}=-30^{\circ}, \phi_{L F}=30^{\circ}$.

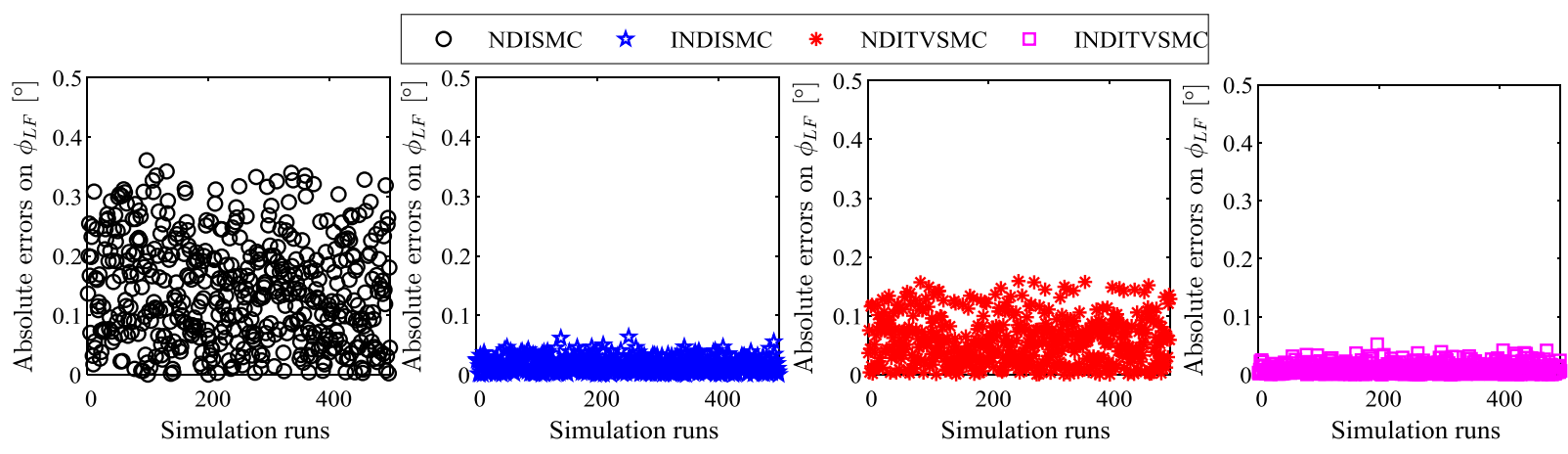

Fig. 22 Distribution of $\phi_{L}$ errors under $A_{z T}=5 \cos (0.3 t), A_{y T}=5 \sin (0.3 t)$ and $\theta_{L F}=-30^{\circ}, \phi_{L F}=30^{\circ}$.

the INDI-SMC and INDI-TVSMC guidance laws. However, all the $\theta_{L}$ errors under the NDI-SMC and NDI-TVSMC guidance laws are around 1.4 and $1 \mathrm{deg}$, respectively. It can be observed from Fig. 22 that most $\phi_{L}$ errors are smaller than 0.05 deg under the INDI-SMC and INDI-TVSMC. The $\phi_{L}$ errors caused by the NDI-SMC and NDITVSMC guidance laws are smaller than 0.4 and 0.2 deg, respectively. In addition, the mean values and standard deviations for the miss distance, terminal LOS angle errors, and terminal LOS rates are listed in Table 3. Note that all the mean values and standard deviations are in acceptable ranges. The terminal LOS rates are around zero, and the mean terminal LOS angle errors by the INDI-SMC and INDITVSMC guidance laws are smaller than that by the NDI-SMC and NDI-TVSMC guidance laws.

In addition, the Monte Carlo results under $A_{z T}=30 \cos (0.3 t)$, $A_{y T}=30 \sin (0.3 t)$ are presented in Figs. 23-25. Figure 23 shows that all the guidance laws are capable of intercepting the target with

Table 3 Statistic results of the Monte Carlo simulations under $A_{z T}=5 \cos (0.3 t)$,

$A_{y T}=5 \sin (0.3 t)$

\begin{tabular}{lcccccc}
\hline \hline Guidance law & Data type & $r_{f}, \mathrm{~m}$ & $\theta_{L}$ errors, deg & $\phi_{L}$ errors, deg & $\dot{\theta}_{L f}, \operatorname{deg} / \mathrm{s}$ & $\dot{\phi}_{L f}, \operatorname{deg} / \mathrm{s}$ \\
\hline NDI-SMC & Mean & 0.8073 & 1.3994 & 0.1414 & 0.0721 & 0.3543 \\
& Standard deviation & 0.1042 & 0.0311 & 0.0880 & 0.2011 & 0.6328 \\
NDI-TVSMC & Mean & 0.8253 & 1.0165 & 0.0574 & 0.0306 & 0.1542 \\
& Standard deviation & 0.0992 & 0.0157 & 0.0398 & 0.4665 & 0.5940 \\
INDI-SMC & Mean & 0.8085 & 0.0159 & 0.0158 & -0.0683 & -0.0108 \\
& Standard deviation & 0.1106 & 0.0129 & 0.0121 & 0.3708 & 0.5438 \\
INDI-TVSMC & Mean & 0.8190 & 0.0188 & 0.0088 & -0.0675 & 0.0633 \\
& Standard deviation & 0.1058 & 0.0156 & 0.0027 & 0.0335 & 0.4970 \\
\hline \hline
\end{tabular}
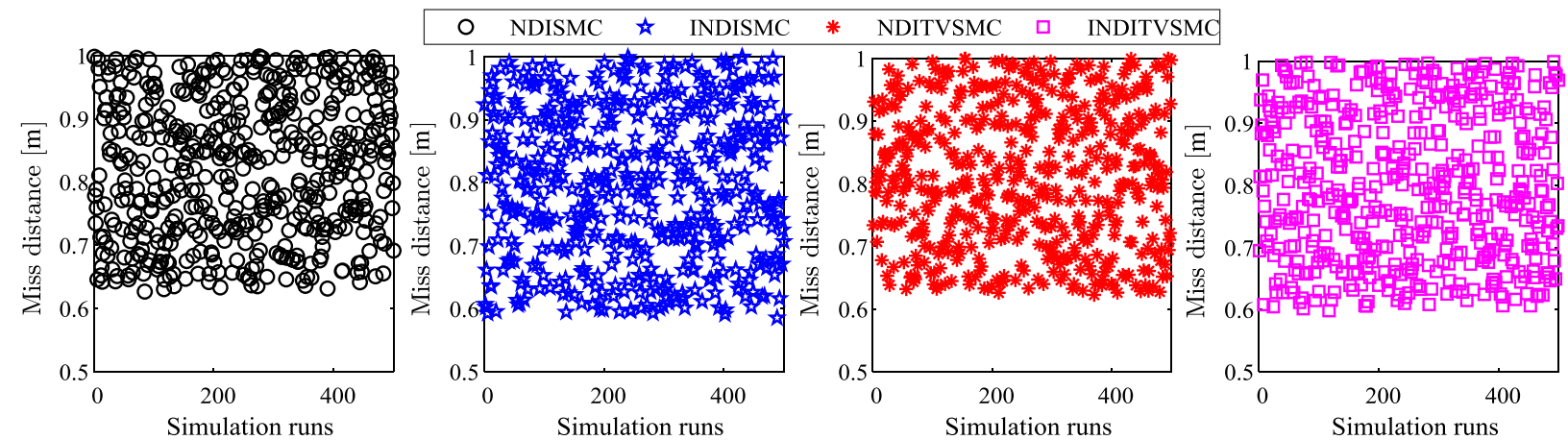

Fig. 23 Miss distance distribution under $A_{z T}=30 \cos (0.3 t), A_{y T}=30 \sin (0.3 t)$ and $\theta_{L F}=-30^{\circ}, \phi_{L F}=30^{\circ}$. 


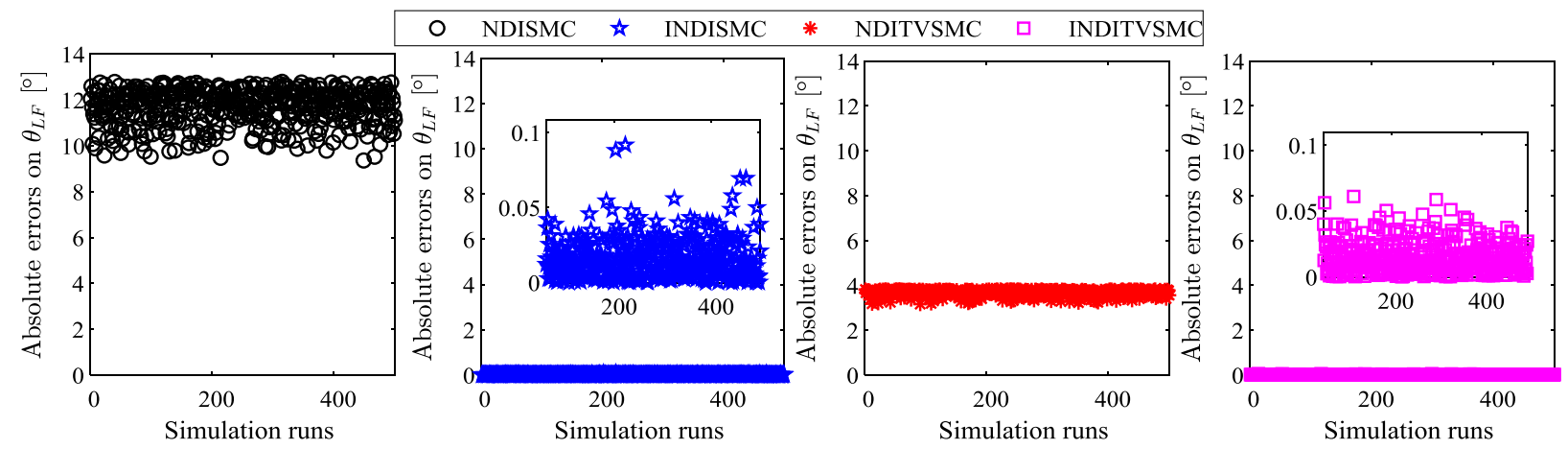

Fig. 24 Distribution of $\theta_{L}$ errors under $A_{z T}=30 \cos (0.3 t), A_{y T}=30 \sin (0.3 t)$ and $\theta_{L F}=-30^{\circ}, \phi_{L F}=30^{\circ}$.

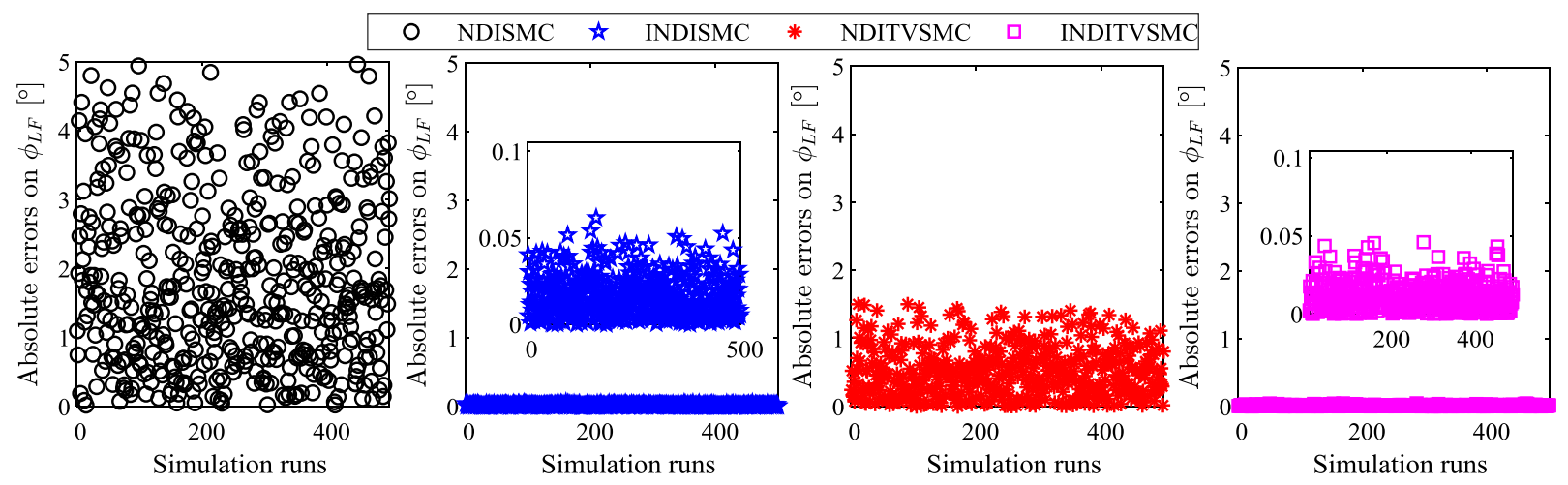

Fig. 25 Distribution of $\phi_{L}$ errors under $A_{z T}=30 \cos (0.3 t), A_{y T}=30 \sin (0.3 t)$ and $\theta_{L F}=-30^{\circ}, \phi_{L F}=30^{\circ}$.

Table 4 Statistic results of the Monte Carlo simulations under $A_{z T}=30 \cos (0.3 t)$, $A_{y T}=30 \sin (0.3 t)$

\begin{tabular}{lcccccc}
\hline \hline Guidance law & Data type & $r_{f}, \mathrm{~m}$ & $\theta_{L}$ errors, deg & $\phi_{L}$ errors, deg & $\dot{\theta}_{L f}, \operatorname{deg} / \mathrm{s}$ & $\dot{\phi}_{L f}$, deg /s \\
\hline NDI-SMC & Mean & 0.8209 & 11.6946 & 1.8514 & 3.0026 & 6.2626 \\
& Standard deviation & 0.1092 & 0.7677 & 1.2455 & 1.0152 & 0.6307 \\
NDI-TVSMC & Mean & 0.8121 & 3.6756 & 0.5172 & 0.2553 & 0.9313 \\
& Standard deviation & 0.1105 & 0.1419 & 0.3629 & 0.1758 & 0.2851 \\
INDI-SMC & Mean & 0.7977 & 0.0160 & 0.0163 & -0.0696 & -0.0855 \\
& Standard deviation & 0.1179 & 0.0129 & 0.0116 & 0.8163 & 0.6906 \\
INDI-TVSMC & Mean & 0.7998 & 0.0147 & 0.0113 & -0.0650 & 0.0891 \\
& Standard deviation & 0.1176 & 0.0114 & 0.0088 & 0.2540 & 0.4460 \\
\hline \hline
\end{tabular}

the miss distance less than $1 \mathrm{~m}$. In Figs. 24 and 25, it can be observed that the $\theta_{L}$ errors and $\phi_{L}$ errors under INDI-SMC and INDI-TVSMC guidance laws are similar to the Monte Carlo results with $A_{z T}=$ $5 \cos (0.3 t), A_{y T}=5 \sin (0.3 t)$. However, all the $\theta_{L}$ errors under the NDI-SMC and NDI-TVSMC guidance laws are around 12 and $4 \mathrm{deg}$, respectively. Meanwhile, most $\phi_{L}$ errors range from 0 to 5 deg and 0 to $1.6 \mathrm{deg}$ under the NDI-SMC and NDI-TVSMC guidance laws. The mean values and standard deviations for the miss distance, terminal LOS angle errors, and terminal LOS rates are listed in Table 4. The terminal LOS rates under the NDI-SMC guidance law are relatively larger than the others. Moreover, the mean values of $\theta_{L}$ errors are not acceptable under the NDI-SMC and NDI-TVSMC guidance laws, whereas small errors are created by the INDI-SMC and INDITVSMC guidance laws.

\section{Conclusions}

The $3 \mathrm{D}$ guidance problem for missiles against maneuvering targets is addressed in this paper, considering coupling effects and terminal LOS angle constraint. The unbounded perturbation near collision caused by target maneuvers is avoided by constructing a sliding variable based on the LOS error dynamics. Two guidance laws using
NDI-SMC and NDI-TVSMC are derived and extended to sensorbased INDI-SMC and INDI-TVSMC guidance laws. The guidance robustness is improved with reduced upper bound of the system perturbation by exploiting the sensor-based guidance laws. The NDI-TVSMC and INDI-TVSMC guidance laws can accelerate the convergence of the LOS tracking errors and avoid large initial sliding variables, as compared with the NDI-SMC and INDI-SMC guidance laws. Compared with the NDI-SMC and NDI-TVSMC guidance laws, the INDI-SMC and INDI-TVSMC guidance laws require smaller gains for perturbation cancellation under the same system uncertainties and target maneuvers. Simulations show that the NDISMC and NDI-TVSMC guidance laws are not feasible against larger target maneuvers. The INDI-SMC can achieve most guidance missions, whereas it is not applicable in handling large initial LOS tracking errors. In contrast, the INDI-TVSMC guidance law achieves all the guidance missions without changing gains in various scenarios. Monte Carlo simulations show that the INDI-SMC and INDI-TVSMC guidance laws can achieve more accurate guidance objectives compared with the NDI-SMC and NDI-TVSMC guidance laws. Although the large perturbations can be rejected by NDI-SMC and NDI-TVSMC with higher gains, undesirable chattering is introduced. 


\section{Acknowledgments}

This work was supported in part by the National Natural Science Foundation of China under Grant 61960206011 and Grant 61633003 , in part by the Beijing Natural Science Foundation under Grant JQ19017, in part by the Project of Science and Technology on Space Intelligent Control Laboratory under Grant 6142208190303, and in part by the Beijing Advanced Discipline Center for Unmanned Aircraft System. The authors would also like to thank the associate editor and reviewers for their valuable comments and constructive suggestions that helped to improve the paper and presentation significantly.

\section{References}

[1] Xin, M., Balakrishnan, S. N., and Ohlmeyer, E. J., "Integrated Guidance and Control of Missiles with Method," IEEE Transactions on Control Systems Technology, Vol. 14, No. 6, 2006, pp. 981-992. https://doi.org/10.1109/TCST.2006.876903

[2] Guo, Y., Hawkins, M., and Wei, B., "Applications of Generalized ZeroEffort-Miss/Zero-Effort-Velocity Feedback Guidance Algorithm," Journal of Guidance, Control, and Dynamics, Vol. 36, No. 3, 2013, pp. 810-820. https://doi.org/10.2514/1.58099

[3] Lu, P., "Theory of Fractional-Polynomial Powered Descent Guidance," Journal of Guidance, Control, and Dynamics, Vol. 43, No. 3, 2020, pp. 398-409. https://doi.org/10.2514/1.G004556

[4] Nesline, F. W., and Zarchan, P., "A New Look at Classical vs Modern Homing Missile Guidance," Journal of Guidance, Control, and Dynamics, Vol. 4, No. 1, 1981, pp. 78-85. https://doi.org/10.2514/3.56054

[5] Zarchan, P., Tactical and Strategic Missile Guidance, 6th ed., AIAA, Reston, VA, 2012, Chap. 2.

[6] Kim, M., and Grider, K., "Terminal Guidance for Impact Attitude Angle Constrained Flight Trajectories," IEEE Transactions on Aerospace and Electronic Systems, Vol. AES-9, No. 6, 1973, pp. 852-859. https://doi.org/10.1109/TAES.1973.309659

[7] Song, T. L., Shin, S. J., and Cho, H., "Impact Angle Control for Planar Engagements," IEEE Transactions on Aerospace and Electronic Systems, Vol. 35, No. 4, 1999, pp. 1439-1444. https://doi.org/10.1109/7.805460

[8] Hattori, Y., Ono, E., and Hosoe, S., "Optimum Vehicle Trajectory Control for Obstacle Avoidance Problem," IEEE Transactions on Mechatronics, Vol. 11, No. 5, 2006, pp. 507-512. https://doi.org/10.1109/TMECH.2006.882981

[9] Harrison, G. A., "Hybrid Guidance Law for Approach Angle and Timeof-Arrival Control," Journal of Guidance, Control, and Dynamics, Vol. 35, No. 4, 2012, pp. 1104-1114. https://doi.org/10.2514/1.56131

[10] Ratnoo, A., and Ghose, D., "Impact Angle Constrained Interception of Stationary Targets," Journal of Guidance, Control, and Dynamics, Vol. 31, No. 6, 2008, pp. 1817-1822. https://doi.org/10.2514/1.37864

[11] Erer, K. S., and Merttopçuoglu, O., "Indirect Impact-Angle-Control Against Stationary Targets Using Biased Pure Proportional Navigation," Journal of Guidance, Control, and Dynamics, Vol. 35, No. 2, 2012, pp. 700-704. https://doi.org/10.2514/1.52105

[12] Lee, C.-H., Kim, T.-H., and Tahk, M.-J., "Interception Angle Control Guidance Using Proportional Navigation with Error Feedback," Journal of Guidance, Control, and Dynamics, Vol. 36, No. 5, 2013, pp. 15561561 . https://doi.org/10.2514/1.58454

[13] Hu, Q., Han, T., and Xin, M., "New Impact Time and Angle Guidance Strategy via Virtual Target Approach," Journal of Guidance, Control, and Dynamics, Vol. 41, No. 8, 2018, pp. 1755-1765. https://doi.org/10.2514/1.G003436

[14] Cho, D., Kim, H. J., and Tahk, M.-J., "Impact Angle Constrained Sliding Mode Guidance Against Maneuvering Target with Unknown Acceleration," IEEE Transactions on Aerospace and Electronic Systems, Vol. 51, No. 2, 2015, pp. 1310-1323. https://doi.org/10.1109/TAES.2015.140358

[15] Zhang, Y., Tang, S., and Guo, J., "An Adaptive Fast Fixed-Time Guidance Law with an Impact Angle Constraint for Intercepting Maneuvering Targets," Chinese Journal of Aeronautics, Vol. 31, No. 6, 2018, pp. 1327-1344.

https://doi.org/10.1016/j.cja.2018.03.017
[16] He, S., Lin, D., and Wang, J., "Continuous Second-Order Sliding Mode Based Impact Angle Guidance Law," Aerospace Science and Technology, Vol. 41, Feb. 2015, pp. 199-208. https://doi.org/10.1016/j.ast.2014.11.020

[17] He, S., Lin, D., and Wang, J., "Robust Terminal Angle Constraint Guidance Law with Autopilot Lag for Intercepting Maneuvering Targets," Nonlinear Dynamics, Vol. 81, No. 1, 2015, pp. 881-892. https://doi.org/10.1007/s11071-015-2037-x

[18] He, S., Song, T., and Lin, D., "Impact Angle Constrained Integrated Guidance and Control for Maneuvering Target Interception," Journal of Guidance, Control, and Dynamics, Vol. 40, No. 10, 2017, pp. 2653 2661. https://doi.org/10.2514/1.G002201

[19] Kumar, S. R., Rao, S., and Ghose, D., "Sliding-Mode Guidance and Control for All-Aspect Interceptors with Terminal Angle Constraints," Journal of Guidance, Control, and Dynamics, Vol. 35, No. 4, 2012. pp. 1230-1246. https://doi.org/10.2514/1.55242

[20] Li, G., Xin, M., and Miao, C., "Finite-Time Input-to-State Stability Guidance Law," Journal of Guidance, Control, and Dynamics, Vol. 41, No. 10, 2018, pp. 2199-2213 https://doi.org/10.2514/1.G003519

[21] Lin, L.-G., and Xin, M., "Missile Guidance Law Based on New Analysis and Design of SDRE Scheme," Journal of Guidance, Control, and Dynamics, Vol. 42, No. 4, 2019, pp. 853-868. https://doi.org/10.2514/1.G003544

[22] He, S., Lee, C.-H., Shin, H.-S., and Tsourdos, A., "Optimal ThreeDimensional Impact Time Guidance with Seeker's Field-of-View Constraint," Chinese Journal of Aeronautics, Vol. 34, No. 2, 2021, pp. $240-251$. https://doi.org/10.1016/j.cja.2020.04.006

[23] Hu, Q., Han, T., and Xin, M., "Analytical Solution for Nonlinear Three-Dimensional Guidance with Impact Angle and Field-of-View Constraints," IEEE Transactions on Industrial Electronics, Vol. 68, No. 4, 2021, pp. 3423-3433. https://doi.org/10.1109/TIE.2020.2982114

[24] Hu, Q., Han, T., and Xin, M., "Three-Dimensional Guidance for Various Target Motions with Terminal Angle Constraints Using Twisting Control," IEEE Transactions on Industrial Electronics, Vol. 67, No. 2, 2020, pp. 1242-1253. https://doi.org/10.1109/TIE.2019.2898607

[25] Liao, Y., Li, H., and Bao, W., "Three-Dimensional Diving Guidance for Hypersonic Gliding Vehicle via Integrated Design of FTNDO and AMSTSMC," IEEE Transactions on Industrial Electronics Vol. 65, No. 3, 2018, pp. 2704-2715. https://doi.org/10.1109/TIE.2017.2736499

[26] Kim, M., Lee, Y., Lee, S., and Kim, Y., "Lyapunov-Based ThreeDimensional Terminal Angle Constrained Guidance Laws," Advances in Aerospace Guidance, Navigation and Control, Springer, Cham, Switzerland, 2015, pp. 39-52. https://doi.org/10.1007/978-3-319-17518-8 3

[27] Lee, Y., and Kim, Y., "Three-Dimensional Impact Angle Control Guidance Law for Missiles Using Dual Sliding Surfaces," IFAC Proceedings Volumes, Vol. 46, No. 19, 2013, pp. 137-142. https://doi.org/10.3182/20130902-5-DE-2040.00075

[28] Park, K. K., Park, S. S., and Ryoo, C. K., "Three-Dimensional Impact Angle Control Guidance Law Using Quaternion," 15th International Conference on Control, Automation and Systems, IEEE Publ., Piscataway, NJ, Oct. 2015, pp. 452-456. https://doi.org/10.1109/ICCAS.2015.7364959

[29] Si, Y., and Song, S., "Three-Dimensional Adaptive Finite-Time Guidance Law for Intercepting Maneuvering Targets," Chinese Journal of Aeronautics, Vol. 30, No. 6, 2017, pp. 1985-2003. https://doi.org/10.1016/j.cja.2017.04.009

[30] Bhaskar, B., Kumar, S. R., and Maity, A., "Three-Dimensional Nonlinear Impact Angle Guidance for Maneuvering Targets," IFACPapersOnLine, Vol. 51, No. 1, 2018, pp. 47-52. https://doi.org/10.1016/j.ifacol.2018.05.009

[31] Wang, X., van Eampen, E.-J., Chu, Q., and Lu, P., "Incremental SlidingMode Fault-Tolerant Flight Control," Journal of Guidance, Control, and Dynamics, Vol. 42, No. 2, 2019, pp. 244-259. https://doi.org/10.2514/1.G003497

[32] Wang, X., Sun, S., van Eampen, E.-J., and Chu, Q., "Quadrotor Fault Tolerant Incremental Sliding Mode Control Driven by Sliding Mode Disturbance Observers," Aerospace Science and Technology, Vol. 87, April 2019, pp. 417-430. https://doi.org/10.1016/j.ast.2019.03.001

[33] Wang, X., van Eampen, E.-J., and Chu, Q., "Quadrotor Fault-Tolerant Incremental Nonsingular Terminal Sliding Mode Control," Aerospace 
Science and Technology, Vol. 95, Dec. 2019, Paper 105514. https://doi.org/10.1016/j.ast.2019.105514

[34] Song, S. H., and Ha, I. J., "A Lyapunov-Like Approach to Performance Analysis of 3-Dimensional Pure PNG Laws," IEEE Transactions on Aerospace and Electronic Systems, Vol. 30, No. 1, 1994, pp. 238-248. https://doi.org/10.1109/7.250424

[35] Hecht, C., "Homing Guidance Using Angular Acceleration of the Line of Sight," AIAA Navigation and Control Conference, AIAA Paper 19912701-CP, Aug. 1991.

https://doi.org/10.2514/6.1991-2701
[36] Kee, P. E., Dong, L., and Siong, C. J., "Near Optimal Midcourse Guidance Law for Flight Vehicle," AIAA Aerospace Sciences Meeting and Exhibit, AIAA Paper 1998-0583, Jan. 1998. https://doi.org/10.2514/6.1998-583

[37] Kumar, S. R., and Ghose, D., "Three-Dimensional Impact Angle Guidance with Coupled Engagement Dynamics," Proceedings of the Institution of Mechanical Engineers, Part G: Journal of Aerospace Engineering, Vol. 231, No. 4, 2017, pp. 621-641. https://doi.org/10.1177/0954410016641442 
2021-07-28

\title{
Sensor-based robust incremental
}

three-dimensional guidance law with

terminal angle constraint

\author{
Han, Tuo
}

AIAA

Han T, Hu Q, Shin H-S, et al., (2021) Sensor-based robust incremental three-dimensional guidance law with terminal angle constraint. Journal of Guidance, Control, and Dynamics, Volume 44, Number 11, November 2021, pp. 2016-2030

https://doi.org/10.2514/1.G006038

Downloaded from Cranfield Library Services E-Repository 